\title{
MATHEMATICAL AND NUMERICAL STUDIES OF NON LINEAR FERROMAGNETIC MATERIALS
}

\author{
PATRICK JOLY ${ }^{1}$ AND OLIVIER VACUS ${ }^{2}$
}

\begin{abstract}
In this paper we are interested in the numerical modeling of absorbing ferromagnetic materials obeying the non-linear Landau-Lifchitz-Gilbert law with respect to the propagation and scattering of electromagnetic waves. In this work we consider the $1 \mathrm{D}$ problem. We first show that the corresponding Cauchy problem has a unique global solution. We then derive a numerical scheme based on an appropriate modification of Yee's scheme, that we show to preserve some important properties of the continuous model such as the conservation of the norm of the magnetization and the decay of the electromagnetic energy. Stability is proved under a suitable CFL condition. Some numerical results for the $1 \mathrm{D}$ model are presented.
\end{abstract}

AMS Subject Classification. 35Q60, 35L60, 65M06.

Received: September 24, 1997. Revised: April 9, 1998 and September 11, 1998.

\section{INTRODUCTION}

\subsection{Exposition of the physical problem}

Without entering into the details of the theory of ferromagnetism (see for this $[2,3]$ or [4]), we briefly describe the physics of our problem.

\subsubsection{The ferromagnetic zone}

By a "ferromagnetic material" we mean a material that possesses a spontaneous magnetization M. (Throughout bold letters will be used for vectors.) Ferromagnetic materials such as ferrites or garnets are widely used in the microwave industry as shifters or circulators for instance. In this paper we study in particular the propagation of electromagnetic waves inside such ferromagnetic materials; they are said to be absorbing materials, we will see what sense we can give to this assertion.

A ferromagnetic zone is a region in which $\mathbf{M} \neq 0$, and we denote by $\Omega$ such a region. This zone will be examined in this paper on a scale large enough to permit the use of a continuous magnetization vector $\mathbf{M}(\mathbf{x})$ at any time; so we do not consider a priori any domain or wall concept [1]. The magnetization $\mathbf{M}$ is assumed to satisfy the Landau-Lifchitz-Gilbert equation (LLG):

$$
\frac{\partial \mathbf{M}}{\partial t}=|\gamma| \mathbf{H}_{T}(\mathbf{H}, \mathbf{M}) \times \mathbf{M}+\frac{\alpha}{|\mathbf{M}|} \mathbf{M} \times \frac{\partial \mathbf{M}}{\partial t},
$$

Keywords and phrases. Maxwell equations, Laundau-Lifchitz-Gilbert equation, Hille-Yosida theorem, FDTD.

1 INRIA Rocquencourt, 78153 Le Chesnay Cedex, France. e-mail: Patrick.Joly@inria.fr

2 Dassault Aviation, 92214 Saint-Cloud Cedex, France. e-mail: olivier.vacus@dassault-aviation.fr 
where $\times$ and $($,$) denote the usual vector and scalar products respectively in \mathbb{R},|\cdot|$ is the usual Euclidean norm, $\gamma$ is the gyromagnetic factor $(\gamma<0)$ and $\alpha$ in the last term is called the damping constant, damping in a sense we shall explain later $(\alpha \geq 0)$.

It is straightforward to show - at least formally - from the (LLG) equation the conservation in time of the norm of the magnetization at any point:

$$
\frac{\partial \mathbf{M}}{\partial t} \cdot \mathbf{M}=\frac{\partial}{\partial t}\left\{|\mathbf{M}|^{2}\right\}=0
$$

Consequently, the LLG equation can be simplified to

$$
\frac{\partial \mathbf{M}}{\partial t}=|\gamma| \mathbf{H}_{T}(\mathbf{H}, \mathbf{M}) \times \mathbf{M}+\frac{\alpha}{\left|\mathbf{M}_{0}\right|} \mathbf{M} \times \frac{\partial \mathbf{M}}{\partial t} \quad \text { where } \quad \mathbf{M}_{0}(\mathbf{x})=\mathbf{M}(\mathbf{x}, t=0) .
$$

\subsubsection{The total magnetic field $\mathbf{H}_{T}(\mathbf{H}, \mathbf{M})$}

The total magnetic field $\mathbf{H}_{T}(\mathbf{H}, \mathbf{M})$ is defined as a sum:

$$
\mathbf{H}_{T}(\mathbf{H}, \mathbf{M})=\mathbf{H}+\mathbf{H}_{\mathrm{eff}}(\mathbf{M})
$$

where $\mathbf{H}$ is the magnetic field appearing in Maxwell's equations

$$
\left\{\begin{array}{c}
\frac{\partial \mathbf{B}}{\partial t}=-\nabla \times \mathbf{E} \\
\varepsilon_{0} \frac{\partial \mathbf{E}}{\partial t}=\nabla \times \mathbf{H}
\end{array}\right.
$$

the relation between $\mathbf{H}, \mathbf{B}$ and $\mathbf{M}$ being

$$
\mathbf{H}=\frac{\mathbf{B}}{\mu_{0}}-\mathbf{M} \text { or } \mathbf{B}=\mu_{0}(\mathbf{H}+\mathbf{M}) \text {. }
$$

In (4) and (5), we denote by:

- B the magnetic induction,

- $\mathbf{E}$ the electric field,

- $\varepsilon_{0}$ and $\mu_{0}$, the electric permittivity and the magnetic permeability in a vacuum.

In this paper we present the non conductive case but no new difficulty would arise if the conductivity $\sigma$ were different from 0.

It is important to note that the (LLG) equation is a non-linear differential equation in time, whereas people often study linear materials in the frequency domain where it is possible to define the magnetic susceptibility $\chi$ and to write that $\mathbf{M}=\chi \mathbf{H}$. In our case, the relation which links $\mathbf{M}$ and $\mathbf{H}$ is implicitly defined by the coupled system (LLG) equation-Maxwell's equations.

The effective field $\mathbf{H}_{\text {eff }}(\mathbf{M})$ is the result of several contributions. In the mode we are interested in, we retain two of them:

$$
\mathbf{H}_{\text {eff }}(\mathbf{M})=\mathbf{H}_{s}+\mathbf{H}_{a}(\mathbf{M})
$$

where $\mathbf{H}_{s}$ is a static field and $\mathbf{H}_{a}(\mathbf{M})$ is a field of anisotropy, a field derived from an energy of anisotropy. In Section 2.1 we define these fields in a ferromagnetic zone $\Omega$. 


\subsubsection{The mathematical model}

As a model of insulating non-linear ferrites, we get the following Cauchy problem from $(2,4,5)$ :

$$
\left\{\begin{array} { l } 
{ \frac { \partial \mathbf { B } } { \partial t } = - \nabla \times \mathbf { E } } \\
{ \varepsilon _ { 0 } \frac { \partial \mathbf { E } } { \partial t } = \nabla \times \mathbf { H } } \\
{ \mathbf { H } = \frac { \mathbf { B } } { \mu _ { 0 } } - \mathbf { M } } \\
{ \frac { \partial \mathbf { M } } { \partial t } = | \gamma | \mathbf { H } _ { T } ( \mathbf { H } , \mathbf { M } ) \times \mathbf { M } + \frac { \alpha } { | \mathbf { M } | } \mathbf { M } \times \frac { \partial \mathbf { M } } { \partial t } }
\end{array} \quad \text { with } \quad \left\{\begin{array}{l}
\mathbf{B}(\mathbf{x}, t=0)=\mathbf{B}_{0}(\mathbf{x}) \\
\mathbf{E}(\mathbf{x}, t=0)=\mathbf{E}_{0}(\mathbf{x}) \\
\mathbf{M}(\mathbf{x}, t=0)=\mathbf{M}_{0}(\mathbf{x})
\end{array}\right.\right.
$$

or, if we simplify the system by eliminating the magnetic induction $\mathbf{B}$,

$$
\left\{\begin{array} { l } 
{ \mu _ { 0 } \frac { \partial \mathbf { H } } { \partial t } + \mu _ { 0 } \frac { \partial \mathbf { M } } { \partial t } = - \nabla \times \mathbf { E } } \\
{ \varepsilon _ { 0 } \frac { \partial \mathbf { E } } { \partial t } = \nabla \times \mathbf { H } } \\
{ \frac { \partial \mathbf { M } } { \partial t } = | \gamma | \mathbf { H } _ { T } ( \mathbf { H } , \mathbf { M } ) \times \mathbf { M } + \frac { \alpha } { | \mathbf { M } | } \mathbf { M } \times \frac { \partial \mathbf { M } } { \partial t } }
\end{array} \quad \text { with } \quad \left\{\begin{array}{l}
\mathbf{H}(\mathbf{x}, t=0)=\mathbf{H}_{0}(\mathbf{x}) \\
\mathbf{E}(\mathbf{x}, t=0)=\mathbf{E}_{0}(\mathbf{x}) \\
\mathbf{M}(\mathbf{x}, t=0)=\mathbf{M}_{0}(\mathbf{x})
\end{array}\right.\right.
$$

\subsection{Presentation of the paper}

Two main mathematical issues naturally arise: 1. Is it possible to show the existence and uniqueness of a solution to this non-linear problem? 2. How can we discretize the equations of this problem to get a numerical modeling of ferromagnetic materials which respects the main properties of the continuous problem?

These are the two questions we intend to address in this paper. Concerning the first point, as far as we know, the only paper where a partial answer is given is Visintin [5] where the existence of weak solutions to a problem very similar to (7) is established. However Visintin includes in $\mathbf{H}_{\text {eff }}(\mathbf{M})$ a new field, derived from an exchange energy, and this new field ensures more regularity for $\mathbf{M}$. His existence result and some secondary properties are proved for the corresponding variational formulation by using a Galerkin method. But, he can not allow $\alpha$ to be 0 , and it is also stated in [5] that "the possible uniqueness of the solution is an open question".

Concerning the second point, the situation is even clearer: we have not found in the literature any satisfying answer to the question of the discretization of the complete non-linear problem (7). All the works on the subject mention at least one of the two following assumptions:

1. the damping is neglected and $\alpha$ is taken to be 0 [13-15];

2. the material is supposed to be saturated and either the internal field is a priori known, or a small signal approximation is made, which leads to a linearization of the equations [10-14].

In the present paper, we establish more complete results concerning both aspects of the problem in the one dimensional case - which is of course a limitation. However all that we do concerning the numerical analysis can be generalized to more space dimensions [16].

We shall show by applying the theory of semi-groups the existence of a unique maximal local strong solution to (7) and then we establish suitable a priori estimates to guarantee that this solution is indeed a maximal global strong solution (the two cases $\alpha=0$ and $\alpha \neq 0$ are treated separately). This theoretical result requiring quite technical proofs, they must be seen as a complementary result to the theorem presented in [17], about existence and uniqueness of weak solutions to (7). (In [17], solutions are sought in $L^{2}(\mathbb{R}) \cap L^{\infty}(\mathbb{R})$ instead of in $H^{1}(\mathbb{R})$ as in the present paper.) In particular, in the case $\alpha \neq 0$, uniqueness will be ensured by results of [17]. 
In the discrete case, we propose an approach based on Yee's scheme [9] and an original way to solve numerically the discrete (LLG) equation. We show that this method ensures the conservation of the norm of the magnetization. We also establish the discrete equivalent of the a priori estimates of the continuous case which guarantees in particular the stability of the scheme under a classical Courant-Friedrichs-Levy condition.

Our paper is organized as follows. In Section 2, we develop the machinery of semi-groups to study the existence and the uniqueness of solutions to the problem (7). In Section 3, we give both time and space discretizations of the equations and their main properties. In Section 4, a representative collection of numerical experiments is presented.

\section{Mathematical Results}

In this section we mainly work from the formulation (8) involving the three vector unknowns $\mathbf{E}, \mathbf{H}$ and $\mathbf{M}$.

\subsection{About the effective field}

In this paragraph we give the precise mathematical definition of the two local fields we consider in our work.

1. The static field $\mathbf{H}_{s}$ is a vector field that is constant in time. For technical reason, $\mathbf{H}_{s}$ is chosen so that :

$$
\mathbf{H}_{s} \in H^{1}(\mathbb{R}),
$$

which does not constitute an actual restriction.

2. The field of anisotropy is derived as follows from the energy of anisotropy $\mathcal{E}_{a}$ - we assume $\mathcal{E}_{a} \in C^{1}\left(L^{2}(\Omega)\right.$; $\left.\mathbb{R}_{+}\right)$. For all $\delta \mathbf{M} \in L^{2}(\Omega)$, we have

$$
\left\langle\mathcal{E}_{a}^{\prime}(\mathbf{M}), \delta \mathbf{M}\right\rangle=-\mu_{0}\left(\mathbf{H}_{a}(\mathbf{M}), \delta \mathbf{M}\right)_{L^{2}}
$$

which ensures that $\mathbf{H}_{a}(\mathbf{M}) \in C^{0}\left(L^{2}(\Omega)^{3} ; L^{2}(\Omega)^{3}\right)$. For simplicity in this paper we consider a special case [1] where

$$
\mathcal{E}_{a}=\mu_{0} \frac{K}{2}|P(\mathbf{M})|^{2}
$$

where $K$ is a positive constant depending on the material and $P(\mathbf{M})$ the projection of $\mathbf{M}$ on the plane perpendicular to the "easy axis" which is simply a privileged direction of the material, not depending on time. Consequently

$$
\mathbf{H}_{a}(\mathbf{M})=-K P(\mathbf{M}) .
$$

Remark 1. If we introduce $\mathbf{p}$, a unit vector along the easy axis, we see that

$$
\mathbf{H}_{a}(\mathbf{M})=-K(\mathbf{M}-(\mathbf{p} \cdot \mathbf{M}) \mathbf{p}) .
$$

This new form will be particularly useful in the numerical part because it leads to

$$
\mathbf{H}_{a}(\mathbf{M}) \times \mathbf{M}=K(\mathbf{p} \cdot \mathbf{M}) \mathbf{p} \times \mathbf{M}
$$

It can be seen in (14) that $\mathbf{H}_{a}(\mathbf{M})$ can be also taken equal to $K(\mathbf{p} \cdot \mathbf{M}) \mathbf{p}$.

As a consequence of these definitions an important property of $\mathbf{H}_{\mathrm{eff}}(\mathbf{M})$ is that it is an affine continuous function of $\mathbf{M}$ - that is to say: $\left[\mathbf{H}_{\mathrm{eff}}(\mathbf{M})-\mathbf{H}_{s}\right]$ is linear in $\mathbf{M}$. 


\subsection{Preliminary results independent of the space dimension}

The results that we give in this paragraph are obtained regardless of both the dimension of the problem and the geometry of the ferromagnetic zone $\Omega$. We denote by $\mathbf{x}=(x, y, z)$ the current point of $\mathbb{R}^{3}$.

\subsubsection{A new form of the ( $L L G)$ equation}

For later use in the mathematical analysis, it would be useful to express $\frac{\partial \mathbf{M}}{\partial t}$ as a function of $\mathbf{M}$ and $\mathbf{H}$. We shall use:

Lemma 1. Given any vector $\mathbf{a}$, the function $A: \mathbf{x} \mapsto \mathbf{x}+\mathbf{a} \times \mathbf{x}$ is a homeomorphism from $\mathbb{R}^{3}$ to $\mathbb{R}^{3}$ and

$$
A^{-1}(\mathbf{y})=\frac{\mathbf{y}+(\mathbf{a} \cdot \mathbf{y}) \mathbf{a}-\mathbf{a} \times \mathbf{y}}{1+|\mathbf{a}|^{2}} .
$$

Moreover, $A^{-1}$ is a contraction.

Proof. Continuity of $A$ is clear. Let $(\mathbf{x}, \mathbf{y}) \in\left(\mathbb{R}^{3}\right)^{2}$ be such that $A(\mathbf{x})=\mathbf{y}$. Taking the scalar product with $\mathbf{a}$, we get $(\mathbf{a} \cdot \mathbf{x})=(\mathbf{a} \cdot \mathbf{y})$, and taking the vector product with $\mathbf{a}$,

$$
\mathbf{a} \times \mathbf{x}+\mathbf{a} \times(\mathbf{a} \times \mathbf{x})=\mathbf{a} \times \mathbf{x}-|\mathbf{a}|^{2} \mathbf{x}+(\mathbf{a} \cdot \mathbf{x}) \mathbf{a}=\mathbf{a} \times \mathbf{y} .
$$

Then we can consider

$$
\left\{\begin{array}{l}
\mathbf{x}+\mathbf{a} \times \mathbf{x}=\mathbf{y} \\
-|\mathbf{a}|^{2} \mathbf{x}+\mathbf{a} \times \mathbf{x}=\mathbf{a} \times \mathbf{y}-(\mathbf{a} \cdot \mathbf{y}) \mathbf{a}
\end{array}\right.
$$

This system is linear in $(\mathbf{x}, \mathbf{a} \times \mathbf{x})$ and can be solved for $\mathbf{x}$ which leads to (15). Finally, taking the scalar product of $A(\mathbf{x})=\mathbf{y}$ with $\mathbf{x}$ gives $|\mathbf{x}|^{2}=\mathbf{x} \cdot \mathbf{y}$. Thus $\forall \mathbf{y},\left|A^{-1}(\mathbf{y})\right| \leq|\mathbf{y}|$.

With $\mathbf{a}=-\frac{\alpha}{|\mathbf{M}|} \mathbf{M}$, the (LLG) equation can be written $A\left(\frac{\partial \mathbf{M}}{\partial t}\right)=|\gamma| \mathbf{H}_{T}(\mathbf{H}, \mathbf{M}) \times \mathbf{M}$. Applying Lemma 1 leads to

$$
\frac{\partial \mathbf{M}}{\partial t}=\frac{|\gamma|}{1+\alpha^{2}}\left[\mathbf{H}_{T}(\mathbf{H}, \mathbf{M}) \times \mathbf{M}+\frac{\alpha}{|\mathbf{M}|} \mathbf{M} \times\left(\mathbf{H}_{T}(\mathbf{H}, \mathbf{M}) \times \mathbf{M}\right)\right]
$$

This new form of the (LLG) equation shows that $\frac{\partial \mathbf{M}}{\partial t}$ is a continuous function of $\mathbf{H}$ and $\mathbf{M}$ that we shall denote by $\mathbf{L}_{G}^{\alpha}$ :

$$
\mathbf{L}_{G}^{\alpha}\left(\mathbf{M}, \mathbf{H}_{T}(\mathbf{H}, \mathbf{M})\right)=\mathbf{H}_{T}(\mathbf{H}, \mathbf{M}) \times \mathbf{M}+\frac{\alpha}{|\mathbf{M}|} \mathbf{M} \times\left(\mathbf{H}_{T}(\mathbf{H}, \mathbf{M}) \times \mathbf{M}\right) .
$$

Remark 2. Using this new form of the (LLG) equation and the Pythagorean theorem (given that $\mathbf{H}_{T} \times \mathbf{M}$ and $\mathbf{M} \times\left(\mathbf{H}_{T} \times \mathbf{M}\right)$ are orthogonal vectors $)$, it is easy to see that

$$
\left|\frac{\partial \mathbf{M}}{\partial t}\right|^{2}=\frac{\gamma^{2}}{1+\alpha^{2}}\left|\mathbf{H}_{T} \times \mathbf{M}\right|^{2} .
$$

We shall also use, in the part devoted to the numerical method

Lemma 2. $\mathbf{L}_{G}^{\alpha}$ is linear with respect to $\mathbf{H}$ and $\mathbf{L}_{G}^{\alpha}(\mathbf{M}, \mathbf{H})=\mathbf{L}_{G}^{\alpha}\left(\mathbf{M}, \frac{\mathbf{B}}{\mu_{0}}\right)$.

Proof. The proof is straightforward. 


\subsubsection{A priori estimates}

We assume in this paragraph that there exists a local solution $(\mathbf{E}, \mathbf{H}, \mathbf{M})$ to problem $(8)$ in $C^{1}\left(0, T ; L^{2}\left(\mathbb{R}^{3}\right)\right)^{3} \cap$ $C^{0}\left(0, T ; H^{1}\left(\mathbb{R}^{3}\right)\right)^{3}$. We assume that the system (8) is satisfied in the classical sense which means that the three equations of (8) are equalities in $C^{0}\left(0, T ; L^{2}\left(\mathbb{R}^{3}\right)\right)$.

\section{- Conservation of the norm of M}

It is straightforward to show - at least formally in our case - from the (LLG) equation the conservation in time of the norm of the magnetization at any point:

$$
\frac{\partial \mathbf{M}}{\partial t} \cdot \mathbf{M}=\frac{\partial}{\partial t}\left\{|\mathbf{M}|^{2}\right\}=0 .
$$

This computation is valid if we assume that for each $\mathbf{x}$, the function $t \mapsto \mathbf{M}(\mathbf{x}, t)$ is of class $C^{1}$, which is not necessary the case under our assumptions. Rigorously we can multiply the (LLG) equation in $L^{2}(\mathbb{R})^{3}$ by $\varphi \mathbf{M}$, with $\varphi \in \mathcal{D}\left(\mathbb{R}^{3}\right)$, to get:

$$
\frac{\mathrm{d}}{\mathrm{d} t}\left(\int_{\mathbb{R}^{3}} \varphi(\mathbf{x})|\mathbf{M}(\mathbf{x}, t)|^{2} \mathrm{~d} \mathbf{x}\right)=0
$$

that is to say

$$
\int_{\mathbb{R}^{3}}\left(|\mathbf{M}(\mathbf{x}, t)|^{2}-\left|\mathbf{M}_{0}(\mathbf{x})\right|^{2}\right) \varphi(\mathbf{x}) \mathrm{d} \mathbf{x}=0
$$

Thus

$$
|\mathbf{M}(\mathbf{x}, t)|^{2}=\left|\mathbf{M}_{0}(\mathbf{x})\right|^{2} \text {, a.e. } \mathbf{x} \in \mathbb{R}^{3} .
$$

In particular $\|\mathbf{M}(t)\|_{L^{2}}=\left\|\mathbf{M}_{0}\right\|_{L^{2}}$ but more generally, if $\mathbf{M}_{0}$ belongs to $L^{p}\left(\mathbb{R}^{3}\right)$, with $1 \leq p \leq+\infty$, then $\mathbf{M}(t)$ belongs to $L^{p}\left(\mathbb{R}^{3}\right)$ for any $t$ and $\|\mathbf{M}(t)\|_{L^{p}}=\left\|\mathbf{M}_{0}\right\|_{L^{p}}$.

\section{- Decay in time of the electromagnetic energy}

The second estimate that we can get in any case expresses the decay in time of the total electromagnetic energy. Multiplying Maxwell's equations by $\mathbf{H}$ and $\mathbf{E}$, we get

$$
\mathbf{E} \cdot(\nabla \times \mathbf{H})-\mathbf{H} \cdot(\nabla \times \mathbf{E})=\varepsilon_{0} \mathbf{E} \cdot \frac{\partial \mathbf{E}}{\partial t}+\mu_{0} \mathbf{H} \cdot \frac{\partial \mathbf{H}}{\partial t}+\mu_{0} \mathbf{H} \cdot \frac{\partial \mathbf{M}}{\partial t} .
$$

We then integrate in space and, using Green's formula, we get

$$
\int_{\mathbb{R}^{3}} \nabla \cdot(\mathbf{E} \times \mathbf{H}) \mathrm{d} \mathbf{x}=0=\int_{\mathbb{R}^{3}}\left(\varepsilon_{0} \mathbf{E} \cdot \frac{\partial \mathbf{E}}{\partial t}+\mu_{0} \mathbf{H} \cdot \frac{\partial \mathbf{H}}{\partial t}\right) \mathrm{d} \mathbf{x}+\mu_{0} \int_{\mathbb{R}^{3}} \mathbf{H} \cdot \frac{\partial \mathbf{M}}{\partial t} \mathrm{~d} \mathbf{x} .
$$

By adding $\mathbf{H}_{\text {eff }}(\mathbf{M})$ to $\mathbf{H}$, we obtain

$$
\frac{\mathrm{d}}{\mathrm{d} t}\left[\frac{\varepsilon_{0}}{2}\|\mathbf{E}\|_{L^{2}}^{2}+\frac{\mu_{0}}{2}\|\mathbf{H}\|_{L^{2}}^{2}\right]-\mu_{0} \int_{\mathbb{R}^{3}} \mathbf{H}_{\mathrm{eff}}(\mathbf{M}) \cdot \frac{\partial \mathbf{M}}{\partial t} \mathrm{~d} \mathbf{x}=-\mu_{0} \int_{\mathbb{R}^{3}} \mathbf{H}_{T}(\mathbf{H}, \mathbf{M}) \cdot \frac{\partial \mathbf{M}}{\partial t} \mathrm{~d} \mathbf{x} .
$$

Let us transform the left hand side of (24). First, concerning the field of anisotropy, we have

$$
-\mu_{0} \int_{\mathbb{R}^{3}}(-K P(\mathbf{M})) \cdot \frac{\partial \mathbf{M}}{\partial t} \mathrm{~d} \mathbf{x}=\frac{\mathrm{d}}{\mathrm{d} t}\left[\frac{\mu_{0} K}{2} \int_{\mathbb{R}^{3}}|P(\mathbf{M})|^{2}\right] .
$$


(In the general case, we would have

$$
\left.\mu_{0} \int_{\mathbb{R}^{3}} \mathbf{H}_{a}(\mathbf{M}) \cdot \frac{\partial \mathbf{M}}{\partial t} \mathrm{~d} \mathbf{x}=\mu_{0}\left(\mathbf{H}_{a}, \frac{\partial \mathbf{M}}{\partial t}\right)_{L^{2}}=-\left\langle\mathcal{E}_{a}^{\prime}(\mathbf{M}), \frac{\partial \mathbf{M}}{\partial t}\right\rangle=-\frac{\mathrm{d}}{\mathrm{d} t}\left(\mathcal{E}_{a}(\mathbf{M})\right) .\right)
$$

Finally, we obtain, using (6) and (25):

$$
\frac{\mathrm{d}}{\mathrm{d} t}\left[\frac{\mu_{0} K}{2}\|P(\mathbf{M})\|_{L^{2}}^{2}-\mu_{0}\left(\mathbf{H}_{s}, \mathbf{M}\right)_{L^{2}}\right]=-\mu_{0} \int_{\mathbb{R}^{3}}\left(\mathbf{H}_{\mathrm{eff}}(\mathbf{M}) \cdot \frac{\partial \mathbf{M}}{\partial t}\right) \mathrm{d} \mathbf{x}
$$

or, by adding some terms constant in time,

$$
\frac{\mathrm{d}}{\mathrm{d} t}\left[\frac{\mu_{0} K}{2}\|P(\mathbf{M})\|_{L^{2}}^{2}+\frac{\mu_{0}}{2}\left\|\mathbf{H}_{s}-\mathbf{M}\right\|_{L^{2}}^{2}\right]=-\mu_{0} \int_{\mathbb{R}^{3}}\left(\mathbf{H}_{\mathrm{eff}}(\mathbf{M}) \cdot \frac{\partial \mathbf{M}}{\partial t}\right) \mathrm{d} \mathbf{x} .
$$

Besides, we have, taking the vector product of the (LLG) equation with $\frac{\partial \mathbf{M}}{\partial t}$ :

$$
\frac{\partial \mathbf{M}}{\partial t} \times \frac{\partial \mathbf{M}}{\partial t}=0=|\gamma|\left(\mathbf{H}_{T}(\mathbf{H}, \mathbf{M}) \cdot \frac{\partial \mathbf{M}}{\partial t}\right) \mathbf{M}-\frac{\alpha}{|\mathbf{M}|}\left|\frac{\partial \mathbf{M}}{\partial t}\right|^{2} \mathbf{M}
$$

that yields, everywhere $\mathbf{M}_{0}$ is different from 0 ,

$$
\mathbf{H}_{T}(\mathbf{H}, \mathbf{M}) \cdot \frac{\partial \mathbf{M}}{\partial t}=\frac{\alpha}{|\gamma|} \frac{1}{|\mathbf{M}|}\left|\frac{\partial \mathbf{M}}{\partial t}\right|^{2} .
$$

On the other hand, everywhere $\mathbf{M}_{0}=0$, this equality reduces to $0=0$, since (18) implies that

$$
\lim _{|\mathbf{M}| \rightarrow 0}\left(\frac{1}{|\mathbf{M}|}\left|\frac{\partial \mathbf{M}}{\partial t}\right|^{2}\right)=0 .
$$

Finally, from $(24,27,29)$, we deduce that

$$
\frac{\mathrm{d}}{\mathrm{d} t}\left[\frac{\varepsilon_{0}}{2}\|\mathbf{E}\|_{L^{2}}^{2}+\frac{\mu_{0}}{2}\|\mathbf{H}\|_{L^{2}}^{2}+\frac{\mu_{0} K}{2}\|P(\mathbf{M})\|_{L^{2}}^{2}+\frac{\mu_{0}}{2}\left\|\mathbf{H}_{s}-\mathbf{M}\right\|_{L^{2}}^{2}\right]=-\mu_{0} \frac{\alpha}{|\gamma|} \int_{\mathbb{R}^{3}} \frac{1}{|\mathbf{M}|}\left|\frac{\partial \mathbf{M}}{\partial t}\right|^{2} \mathrm{~d} x .
$$

It can be understood with this equality why the sign of $\alpha$ is crucial and why it is called a damping constant. It also explains why the ferromagnetic materials are expected to be absorbing since it shows that the electromagnetic energy

$$
\mathcal{E}_{T}(\mathbf{E}, \mathbf{H}, \mathbf{M})=\frac{\varepsilon_{0}}{2}\|\mathbf{E}\|_{L^{2}}^{2}+\frac{\mu_{0}}{2}\|\mathbf{H}\|_{L^{2}}^{2}+\frac{\mu_{0} K}{2}\|P(\mathbf{M})\|_{L^{2}}^{2}+\frac{\mu_{0}}{2}\left\|\mathbf{H}_{s}-\mathbf{M}\right\|_{L^{2}}^{2}
$$

is decreasing in time. From (31), we get

$$
\begin{aligned}
& \varepsilon_{0}\|\mathbf{E}(t)\|_{L^{2}}^{2} \leq\left(\varepsilon_{0}\left\|\mathbf{E}_{0}\right\|_{L^{2}}^{2}+\mu_{0}\left\|\mathbf{H}_{0}\right\|_{L^{2}}^{2}+(1+K) \mu_{0}\left\|\mathbf{M}_{0}\right\|_{L^{2}}^{2}+\mu_{0}\left\|\mathbf{H}_{s}\right\|_{L^{2}}^{2}\right) \\
& \mu_{0}\|\mathbf{H}(t)\|_{L^{2}}^{2} \leq\left(\varepsilon_{0}\left\|\mathbf{E}_{0}\right\|_{L^{2}}^{2}+\mu_{0}\left\|\mathbf{H}_{0}\right\|_{L^{2}}^{2}+(1+K) \mu_{0}\left\|\mathbf{M}_{0}\right\|_{L^{2}}^{2}+\mu_{0}\left\|\mathbf{H}_{s}\right\|_{L^{2}}^{2}\right)
\end{aligned} .
$$

These estimates show that $\mathbf{E} \in L^{2}\left(\mathbb{R}^{3}\right)^{3}$ and $\mathbf{H} \in L^{2}\left(\mathbb{R}^{3}\right)^{3}$ as soon as $\mathbf{E}_{0} \in L^{2}\left(\mathbb{R}^{3}\right)^{3}$ and $\mathbf{H}_{0} \in L^{2}\left(\mathbb{R}^{3}\right)^{3}$ (and of course $\left.\mathbf{M}_{0} \in L^{2}\left(\mathbb{R}^{3}\right)^{3}\right)$. 


\subsection{The 1D model}

In order to shorten the forthcoming formulae in our mathematical developments, henceforth all the positive physical constants except $\alpha-\varepsilon_{0}, \mu_{0},|\gamma|, K$ and $1+\alpha^{2}$ - will be replaced by one.

Moreover we consider from now on a mono-dimensional problem: we assume that all the unknowns in the following problem depend only on $\mathrm{x}$, the first space variable, and $t$, the time variable. In other words we are interested in plane waves propagating along the $x$-direction. We shall denote by $\left(\mathbf{e}_{x}, \mathbf{e}_{y}, \mathbf{e}_{z}\right)$ the orthonormal basis of $\mathbb{R}^{3}$ where $\mathbf{e}_{x}$ is parallel to the $x$-direction.

The first consequence of this 1D hypothesis is to simplify the definition of the curl operator. Given any field $\mathbf{A}=\left(A_{x}, A_{y}, A_{z}\right)$ depending only on the $x$ variable, we simply have

$$
\nabla \times \mathbf{A}=\left(0,-\frac{\partial A_{z}}{\partial x}, \frac{\partial A_{y}}{\partial x}\right)
$$

The interest of this remark appears if you notice that taking the projection of (4) on the propagation axis leads to

$$
\frac{\mathrm{d} B_{x}}{\mathrm{~d} t}=0 \quad \text { and } \quad \frac{\mathrm{d} E_{x}}{\mathrm{dt}}=0
$$

and so, the longitudinal components $E_{x}$ and $B_{x}$ are both constant in the 1D problem. (Concerning the magnetic field, we simply have $H_{x}=-M_{x}$ up to a constant.) However we would like to underline the fact that, contrary to what happens in the vacuum, it is not possible - even in the $1 \mathrm{D}$ case - to consider the propagation of a transverse electric mode on the one hand and the propagation of a transverse magnetic mode on the other hand because all the components of $\mathbf{H}$ are coupled by the (LLG) equation.

We shall also use in the sequel the two following properties of $\mathbf{L}_{G}^{\alpha}$ in the $1 \mathrm{D}$ case:

Lemma 3. Assuming that $\mathbf{H}_{\text {s }}$ belongs to $L^{2}(\mathbb{R})^{3}, \mathbf{L}_{G}^{\alpha}$ maps $L^{2}(\mathbb{R})^{3} \cap L^{\infty}(\mathbb{R})^{3} \times L^{2}(\mathbb{R})^{3}$ into $L^{2}(\mathbb{R})^{3}$.

Proof. We can deduce from (3) that

$$
\left\|\mathbf{H}_{T}(\mathbf{H}, \mathbf{M}) \times \mathbf{M}\right\|_{L^{2}} \leq\|\mathbf{M}\|_{L^{\infty}}\|\mathbf{H}\|_{L^{2}}+\|\mathbf{M}\|_{L^{\infty}}\left\|\mathbf{H}_{s}\right\|_{L^{2}}+C\|\mathbf{M}\|_{L^{\infty}}\|\mathbf{M}\|_{L^{2}}
$$

and then

$$
\left\|\mathbf{L}_{G}^{\alpha}\left(\mathbf{M}, \mathbf{H}_{T}(\mathbf{H}, \mathbf{M})\right)\right\|_{L^{2}} \leq(1+\alpha)\|\mathbf{M}\|_{L^{\infty}}\left(\|\mathbf{H}\|_{L^{2}}+\left\|\mathbf{H}_{s}\right\|_{L^{2}}+C\|\mathbf{M}\|_{L^{2}}\right)
$$

Lemma 4. Assuming that $\mathbf{H}_{s}$ belongs to $H^{1}(\mathbb{R})^{3}, \mathbf{L}_{G}^{\alpha}$ is locally Lipchitz from $H^{1}(\mathbb{R})^{3} \times H^{1}(\mathbb{R})^{3}$ into $H^{1}(\mathbb{R})^{3}$ if and only if $\alpha=0$.

Proof. If $\alpha=0$, the result is straightforward since the map $(u, v) \rightarrow u v$ is locally Lipschitz from $H^{1}(\mathbb{R}) \times H^{1}(\mathbb{R})$ to $H^{1}(\mathbb{R})$.

To understand what happens if $\alpha \neq 0$, the reader can check that the "absolute value" map is not locally Lipchitz from $H^{1}(I)$ to $H^{1}(I)$, for any open interval $I$, by considering the two functions $u_{n}=2+\sin (n x)$ and $v_{n}=-2+\sin (n x)$. Anyway this case is not important for our paper. 


\subsection{Local results}

The aim of this paragraph is to show the existence of a unique maximal local strong solution by applying the following theorem (see [6] or [7]) which applies to semi-linear abstract evolution equations:

Theorem 1. Let $\mathcal{H}$ be a Banach space and $A$ a maximal monotone operator [6] with domain $D(A)$. Let $F$ : $\mathcal{H} \rightarrow \mathcal{H}$ be a locally Lipschitz function on $D(A)$. Then, for all $u_{0} \in D(A)$, there exists a unique solution to

$$
\left\{\begin{array}{l}
\frac{\partial u}{\partial t}=A u+F(u) \\
u(t=0)=u_{0}
\end{array}\right.
$$

with $u \in C^{1}\left(0, T_{\max } ; D(A)\right) \cap C^{0}\left(0, T_{\max } ; \mathcal{H}\right)$ where either

$$
\left(T_{\max }=+\infty\right) \text { or }\left(T_{\max }<+\infty \text { and } \lim _{t \nearrow T_{\max }}\|u\|_{\mathcal{H}}=+\infty\right)
$$

In order to apply this theorem, it is natural to try to divide our problem into the linear part - the operator $A$ - and the non-linear part - the function $F$, and then to check the required assumptions. It is exactly what is done in the next paragraph in the case $\alpha=0$. But in the general case $(\alpha \neq 0)$, the function $F$ (indeed the function $\mathbf{L}_{G}^{\alpha}$ ) is no longer locally Lipchitz as mentioned in Lemma 4 and we have to adapt our proof.

Remark 3. It would have been possible to treat the general case in the same manner as the case $\alpha=0$ if we had considered (37), the same equation as in [5], where the term $1 /|\mathbf{M}|$ is omitted:

$$
\frac{\partial \mathbf{M}}{\partial t}=|\gamma| \mathbf{H} \times \mathbf{M}+\alpha \mathbf{M} \times(\mathbf{H} \times \mathbf{M}) .
$$

But this change in the LLG equation is valid only if the norm of the magnetization can be assumed to be constant in space, which is impossible when you consider the interface between the vacuum and a ferromagnetic material.

We establish below local results by applying Theorem 1. We consider separately the two cases $\alpha=0$ and $\alpha \neq 0$, that is to say with and without damping. In each case, we shall decompose our proof into three steps. First we define the appropriate functional framework $\mathcal{H}$ as well as the operator $A$ and the non-linear part $F$. Then we establish that $A$, and $F$, have the required properties.

\subsubsection{The case $\alpha=0$}

The (LLG) equation is

$$
\frac{\partial \mathbf{M}}{\partial t}=\mathbf{H}_{T}(\mathbf{H}, \mathbf{M}) \times \mathbf{M}
$$

\section{- Mathematical framework}

We introduce $\mathcal{H}=L^{2}(\mathbb{R})^{3} \times L^{2}(\mathbb{R})^{3} \times H^{1}(\mathbb{R})^{3}$, which is clearly a Hilbert space, and take as our unknown the vector $\mathbf{u}=(\mathbf{E}, \mathbf{H}, \mathbf{M}) \in \mathcal{H}$. We also introduce the Hilbert space $H(\operatorname{curl}, \mathbb{R})=\left\{v \in L^{2}(\mathbb{R})^{3} ; \operatorname{curl}(v) \in L^{2}(\mathbb{R})^{3}\right\}$, which is in the $1 \mathrm{D}$ case nothing else than $L^{2}(\mathbb{R}) \times H^{1}(\mathbb{R}) \times H^{1}(\mathbb{R})$. It is now possible to rewrite the three equations 
of (8) as:

$$
\left\{\begin{array}{l}
\frac{\partial \mathbf{E}}{\partial t}=\text { curl } \mathbf{H} \\
\frac{\partial \mathbf{H}}{\partial t}+\frac{\partial \mathbf{M}}{\partial t}=- \text { curl } \mathbf{E} \\
\frac{\partial \mathbf{M}}{\partial t}=\mathbf{H}_{T} \times \mathbf{M} \quad\left(\text { with } \mathbf{H}_{T}=\mathbf{H}+\mathbf{H}_{s}+\mathbf{H}_{a}(\mathbf{M})\right)
\end{array}\right.
$$

that leads to the following system of evolution equations:

$$
\left\{\begin{aligned}
\frac{\partial \mathbf{E}}{\partial t} & =\operatorname{curl} \mathbf{H} \\
\frac{\partial \mathbf{H}}{\partial t} & =-\operatorname{curl} \mathbf{E}-\mathbf{H}_{T}(\mathbf{H}, \mathbf{M}) \times \mathbf{M} \\
\frac{\partial \mathbf{M}}{\partial t} & =\mathbf{H}_{T}(\mathbf{H}, \mathbf{M}) \times \mathbf{M}
\end{aligned}\right.
$$

It is now natural to introduce the unbounded operator

$$
A(\mathbf{u})=A(\mathbf{E}, \mathbf{H}, \mathbf{M})=(\operatorname{curl} \mathbf{H},-\operatorname{curl} \mathbf{E}, 0)
$$

with domain $D(A)=H(\operatorname{curl}, \mathbb{R}) \times H(\operatorname{curl}, \mathbb{R}) \times H^{1}(\mathbb{R})^{3}$, and the function:

$$
F(\mathbf{u})=F(\mathbf{E}, \mathbf{H}, \mathbf{M})=\left(0,-\mathbf{H}_{T}(\mathbf{H}, \mathbf{M}) \times \mathbf{M}, \mathbf{H}_{T}(\mathbf{H}, \mathbf{M}) \times \mathbf{M}\right)
$$

With these notations, the problem (8) is equivalent to:

$$
\left\{\begin{array}{l}
\frac{\partial \mathbf{u}}{\partial t}=A \mathbf{u}+F(\mathbf{u}) \\
\mathbf{u}(t=0)=\mathbf{u}_{0}
\end{array}\right.
$$

where of course $\mathbf{u}_{0}=\left(\mathbf{E}_{0}, \mathbf{H}_{0}, \mathbf{M}_{0}\right)$. It remains to show that $A$ is maximal monotone and that $F$ is locally Lipschitz from $D(A)$ to $D(A)$.

- $A$ is a maximal monotone operator

This result is not surprising since $A$ is nothing other than the operator associated to Maxwell's equations. First using the Green's formula:

$$
\int_{\mathbb{R}}(\operatorname{curl} \mathbf{E} \cdot \mathbf{H}-\operatorname{curl} \mathbf{H} \cdot \mathbf{E}) \mathrm{d} x=0
$$

for all $(\mathbf{E}, \mathbf{H})$ in $H(\operatorname{curl}, \mathbb{R}) \times H(\operatorname{curl}, \mathbb{R})$ yields to:

$$
(A \mathbf{u}, \mathbf{u})_{\mathcal{H}}=0, \quad \forall \mathbf{u} \in D(A)
$$


Then, to get the surjectivity of $A+I$ from $D(A)$ in $\mathcal{H}$, we have to show that, for all $(\mathbf{e}, \mathbf{h}, \mathbf{m})$ in $\mathcal{H}$, there exists $(\mathbf{E}, \mathbf{H}, \mathbf{M})$ in $D(A)$ such that:

$$
\left\{\begin{array}{l}
-\operatorname{curl} \mathbf{H}+\mathbf{E}=\mathbf{e} \\
\operatorname{curl} \mathbf{E}+\mathbf{H}=\mathbf{h} \\
\mathbf{M}=\mathbf{m}
\end{array}\right.
$$

Reminding that the longitudinal components are constant, this leads to solve for the transverse components

$$
\operatorname{curl}(\operatorname{curl} \mathbf{E})+\mathbf{E}=\mathbf{e}+\operatorname{curlh}
$$

The Lax-Milgram theorem ensures that there exists a unique solution $H^{1}(\mathbb{R})^{2}$ - here are considered only the transverse components - for a right hand side in $L^{2}(\mathbb{R})^{2}$. This implies in our case that $\mathbf{E}$ is in $H(\operatorname{curl}, \mathbb{R})$ for $(\mathbf{e}, \mathbf{h})$ in $L^{2}(\mathbb{R})^{3}$. We can then conclude with $\mathbf{H}=\mathbf{h}-\operatorname{curl} \mathbf{E}$.

- $F$ is a locally Lipschitz continuous function from $D(A)$ to $D(A)$

It is clear by Lemma 4 .

This was the last point of our proof. Eventually we can state the

Theorem 2. Given $\left(\mathbf{E}_{0}, \mathbf{H}_{0}, \mathbf{M}_{0}\right) \in H(\operatorname{curl}, \mathbb{R}) \times H(\operatorname{curl}, \mathbb{R}) \times H^{1}(\mathbb{R})^{3}$, there exists a unique local maximal strong solution of (8) so that:

$$
\left\{\begin{array}{l}
\mathbf{E} \in C^{1}\left(0, T_{\max } ; L^{2}(\mathbb{R})^{3}\right) \cap C^{0}\left(0, T_{\max } ; H(\operatorname{curl}, \mathbb{R})\right) \\
\mathbf{H} \in C^{1}\left(0, T_{\max } ; L^{2}(\mathbb{R})^{3}\right) \cap C^{0}\left(0, T_{\max } ; H(\operatorname{curl}, \mathbb{R})\right) \\
\mathbf{M} \in C^{1}\left(0, T_{\max } ; H^{1}(\mathbb{R})^{3}\right)
\end{array}\right.
$$

with moreover the following alternative: either $T_{\max }=+\infty$,

$$
\text { or } \lim _{t \nearrow T_{\max }}\left\{\|\mathbf{E}(t)\|_{H(\text { curl })}^{2}+\|\mathbf{H}(t)\|_{H(\text { curl })}^{2}+\|\mathbf{M}(t)\|_{H^{1}}^{2}\right\}=+\infty .
$$

\subsubsection{The case $\alpha \neq 0$}

The (LLG) equation is

$$
\frac{\partial \mathbf{M}}{\partial t}=\mathbf{H}_{T}(\mathbf{H}, \mathbf{M}) \times \mathbf{M}+\frac{\alpha}{|\mathbf{M}|} \mathbf{M} \times\left(\mathbf{H}_{T}(\mathbf{H}, \mathbf{M}) \times \mathbf{M}\right)=\mathbf{L}_{G}^{\alpha}\left(\mathbf{M}, \mathbf{H}_{T}(\mathbf{H}, \mathbf{M})\right) .
$$

As said in the introduction, in the case $\alpha \neq 0$, we shall only present an existence result but, since

$$
H^{1}(\mathbb{R}) \subset L^{2}(\mathbb{R}) \cap L^{\infty}(\mathbb{R})
$$

uniqueness will be ensured by the theorem about weak solutions presented in [17]. (For this reason, this paragraph can be seen as complementary to [17].) 
First of all it must be said that this existence result is obtained under a new and purely technical assumption on the initial distribution of magnetization $\mathbf{M}_{0}$, namely

$$
\frac{\mathbf{M}_{0}}{\left|\mathbf{M}_{0}\right|} \in H^{1}(\mathbb{R})
$$

or, to be more rigorous,

$$
\exists \mathbf{m}_{0} \in H^{1}(\mathbb{R}) \text { such that: } \forall x \in \mathbb{R},\left|\mathbf{M}_{0}(x)\right| \neq 0 \Rightarrow \frac{\mathbf{M}_{0}(x)}{\left|\mathbf{M}_{0}(x)\right|}=\mathbf{m}_{0}(x) .
$$

However it is important to understand that assumption (53) represents no serious restriction, other than $\mathbf{M}_{0}$ must be of bounded support (see Rem. 4).

- A new formulation

We know that it is possible to write the three equations of (8) as the following system of evolution equations:

$$
\left\{\begin{array}{l}
\frac{\partial \mathbf{E}}{\partial t}=\operatorname{curl} \mathbf{H} \\
\frac{\partial \mathbf{H}}{\partial t}=-\operatorname{curl} \mathbf{E}-\mathbf{L}_{G}^{\alpha}\left(\mathbf{M}, \mathbf{H}_{T}(\mathbf{H}, \mathbf{M})\right) \\
\frac{\partial \mathbf{M}}{\partial t}=\mathbf{L}_{G}^{\alpha}\left(\mathbf{M}, \mathbf{H}_{T}(\mathbf{H}, \mathbf{M})\right)
\end{array}\right.
$$

associated with the initial data $\left(\mathbf{E}_{0}, \mathbf{H}_{0}, \mathbf{M}_{0}\right)$. The idea of this section is to consider a normalized magnetization vector $\mathbf{m}$; more exactly, we define the following problem

$$
\left\{\begin{array}{l}
\frac{\partial \mathbf{E}}{\partial t}=\operatorname{curl} \mathbf{H} \\
\frac{\partial \mathbf{H}}{\partial t}=-\operatorname{curl} \mathbf{E}-\left|\mathbf{M}_{0}\right| \mathbf{L}^{\alpha}(\mathbf{m}, \mathbf{H}) \\
\frac{\partial \mathbf{m}}{\partial t}=\mathbf{L}^{\alpha}(\mathbf{m}, \mathbf{H})
\end{array}\right.
$$

where

$$
\mathbf{L}^{\alpha}\left(\mathbf{m}, \mathbf{H}_{T}\right)=\left[\mathbf{H}_{T}\left(\mathbf{H},\left|\mathbf{M}_{0}\right| \mathbf{m}\right) \times \mathbf{m}+\alpha \mathbf{m} \times\left(\mathbf{H}_{T}\left(\mathbf{H},\left|\mathbf{M}_{0}\right| \mathbf{m}\right) \times \mathbf{m}\right)\right]
$$

associated with the initial data $\left(\mathbf{E}_{0}, \mathbf{H}_{0}, \mathbf{m}_{0}\right)$ with $\mathbf{m}_{0}=\frac{\mathbf{M}_{0}}{\left|\mathbf{M}_{0}\right|} \in H^{1}(\mathbb{R})$ as indicated in assumption (53). Now let $(\mathbf{E}, \mathbf{H}, \mathbf{m})$ be a solution to problem (55) and let $\mathbf{M}(x, t)=\left|\mathbf{M}_{0}(x)\right| \mathbf{m}(x, t)$. We have $\mathbf{M} \in H^{1}(\Omega)$, and also, because of the conservation of the norm of $\mathbf{M}$ (and $\mathbf{m}$ ):

$$
\mathbf{L}_{G}^{\alpha}(\mathbf{M}, \mathbf{H})=\left|\mathbf{M}_{0}\right| \mathbf{L}^{\alpha}(\mathbf{m}, \mathbf{H})
$$

and

$$
\frac{\partial \mathbf{M}}{\partial t}=\mathbf{L}_{G}^{\alpha}(\mathbf{M}, \mathbf{H}) \Leftrightarrow \frac{\partial \mathbf{m}}{\partial t}=\mathbf{L}^{\alpha}(\mathbf{m}, \mathbf{H})
$$

In other words every solution to problem (55) is clearly a solution to problem (54), and it only remains now to conclude to apply Theorem 1 to problem (55). 
- Mathematical framework

The notation is the same as in the case $\alpha=0$. We take as unknown the vector $\mathbf{u}=(\mathbf{E}, \mathbf{H}, \mathbf{m}) \in \mathcal{H}$. Again we introduce the operator

$$
A(\mathbf{u})=A(\mathbf{E}, \mathbf{H}, \mathbf{m})=(\operatorname{curl} \mathbf{H},-\operatorname{curl} \mathbf{E}, 0)
$$

with domain $D(A)=H(\operatorname{curl}, \Omega) \times H(\operatorname{curl}, \Omega) \times H^{1}(\Omega)^{3}$, and the function:

$$
F_{\mathbf{M}_{0}}(\mathbf{u})=\left(0,-\left|\mathbf{M}_{0}\right| \mathbf{L}^{\alpha}\left(\mathbf{m}, \mathbf{H}_{T}\right), \mathbf{L}^{\alpha}\left(\mathbf{m}, \mathbf{H}_{T}\right)\right)
$$

With this notation, the problem (55) is equivalent to:

$$
\left\{\begin{array}{l}
\frac{\partial \mathbf{u}}{\partial t}=A \mathbf{u}+F_{\mathbf{M}_{0}}(\mathbf{u}) \\
\mathbf{u}(t=0)=\mathbf{u}_{0}
\end{array}\right.
$$

with $\mathbf{u}_{0}=\left(\mathbf{E}_{0}, \mathbf{H}_{0}, \mathbf{m}_{0}\right)$. It remains to see that

- $A$ is a maximal monotone operator

It is clear since this operator is exactly the same as in the previous section.

- $F_{\mathrm{M}_{0}}$ is a locally Lipschitz continuous function from $D(A)$ to $D(A)$

First it is obvious that $F_{\mathbf{M}_{0}}$ maps $D(A)$ to $D(A)$ since $H^{1}(\Omega)^{3}$ is an algebra. Moreover $F_{\mathbf{M}_{0}}$ is locally Lipchitz by Lemma 4 .

Applying Theorem 1, we obtain a local solution $(\mathbf{E}, \mathbf{H}, \mathbf{m})$ to problem (55) ; it is then possible to define $\mathbf{M}=\left|\mathbf{M}_{0}\right| \mathbf{m}$ a solution to problem (54) and finally we can state

Theorem 3. Given $\left(\mathbf{E}_{0}, \mathbf{H}_{0}, \mathbf{M}_{0}\right) \in H(\operatorname{curl}, \Omega) \times H(\operatorname{curl}, \Omega) \times H^{1}(\Omega)^{3}$, there exists a unique local maximal strong solution of (8) so that:

$$
\left\{\begin{array}{l}
\mathbf{E} \in C^{1}\left(0, T_{\max } ; L^{2}(\Omega)^{3}\right) \cap C^{0}\left(0, T_{\max } ; H(\operatorname{curl}, \Omega)\right) \\
\mathbf{H} \in C^{1}\left(0, T_{\max } ; L^{2}(\Omega)^{3}\right) \cap C^{0}\left(0, T_{\max } ; H(\operatorname{curl}, \Omega)\right) \\
\mathbf{M} \in C^{1}\left(0, T_{\max } ; H^{1}(\Omega)^{3}\right)
\end{array}\right.
$$

with the following alternative: either $T_{\max }=+\infty$,

$$
\text { or } \lim _{t \nearrow T_{\max }}\left\{\|\mathbf{E}(t)\|_{H(\text { curl })}^{2}+\|\mathbf{H}(t)\|_{H(\text { curl })}^{2}+\|\mathbf{M}(t)\|_{H^{1}}^{2}\right\}=+\infty
$$

Remark 4. Without studying the most general case, let us see briefly that there exists a large class of admissible initial distributions. For instance it suffices that $\mathbf{M}_{0}=f(x) \mathbf{u}$ with $\mathbf{u}$ a fixed vector and $f \in H^{1}(\mathbb{R})$ a positive function with bounded support: it is easy in this case to construct $\mathbf{m}_{0} \in H^{1}(\mathbb{R})$ equal to $\mathbf{u}$ on the support of $f$. 


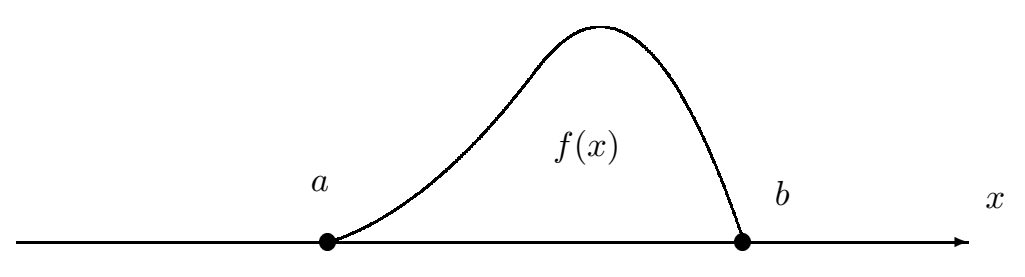

$$
\mathbf{M}_{0}=0 \quad \mathbf{M}_{0} \neq 0 \quad \mathbf{M}_{0}=0
$$

From this first example, the reader can easily check that more generally, one may consider any ferromagnetic zone $[a, b]$ such that

$$
\begin{cases}\left.\exists \varepsilon_{a}>0, \exists \mathbf{u}_{a} \in \mathbb{R}^{3}, \forall x \in\right] a, a+\varepsilon_{a}\left[, \mathbf{M}_{0}=f_{a}(x) \mathbf{u}_{a}\right. & \left(f_{a}(x) \geq 0\right) \\ \left.\exists \varepsilon_{b}>0, \exists \mathbf{u}_{b} \in \mathbb{R}^{3}, \forall x \in\right] b-\varepsilon_{b}, b\left[, \mathbf{M}_{0}=f_{b}(x) \mathbf{u}_{b}\right. & \left(f_{b}(x) \geq 0\right) .\end{cases}
$$

In this case, we have

$$
\left(\frac{\mathbf{M}_{0}}{\left|\mathbf{M}_{0}\right|}\right)_{] a, a+\varepsilon_{a}[} \in H^{1}(] a, a+\varepsilon_{a}[)^{3} \text { and }\left(\frac{\mathbf{M}_{0}}{\left|\mathbf{M}_{0}\right|}\right)_{] b-\varepsilon_{b}, b[} \in H^{1}(] b-\varepsilon_{b}, b[)^{3}
$$

and it is easy to check, because $\exists C_{\varepsilon}>0$ such that $\left|\mathbf{M}_{0}\right| \geq C_{\varepsilon}$ on $] a+\varepsilon_{a}, b-\varepsilon_{b}[$, that

$$
\left.\frac{\mathbf{M}_{0}}{\left|\mathbf{M}_{0}\right|}\right] a+\varepsilon_{a}, b-\varepsilon_{b}\left[\in H^{1}(] a+\varepsilon_{a}, b-\varepsilon_{b}[)^{3} .\right.
$$

Hence the result since $\mathbf{M}_{0}$ is continuous for $x=a+\varepsilon_{a}$ and $x=b-\varepsilon_{b}$. And of course, the ferromagnetic zone can be defined as the union of such disjoint intervals:

$$
\Omega=\bigcup_{i}\left[a_{i}, b_{i}\right]
$$

which leads to a large class of admissible initial distributions.

On the contrary $\mathbf{M}_{0}=\mathrm{e}^{-x^{2}} \sin (x) \mathbf{u}$ is not an admissible initial data.

As a conclusion let us simply say that assumption (53) only implies a kind of control on the way $\mathbf{M}_{0}$ vanishes to zero.

It remains now to show that the local solutions of Theorem 2 and Theorem 3 are indeed global. Rather than expliciting two very similar demonstrations, we now end this mathematical part of our work in the most general case: no assumption is made either on $\alpha$ or on $\mathbf{M}_{0}$. This way we can eventually conclude in each case.

\subsection{A new estimate for $\mathbf{H}$}

We have now to verify that no local solution can blow up in a finite time. With (21) and (33) which are valid in our case for any $T<T_{\max }$, only the $L^{2}$ norm of the local solutions provided by Theorem 2 is controlled. So, to prove global existence we have to find $H^{1}$ estimates for these solutions depending on $t$ in a locally bounded manner. More precisely we first establish in this paragraph that $\mathbf{H} \in H$ (curl, $\mathbb{R})$.

Before beginning, let us introduce the following technical lemma. 
Lemma 5. Concerning the infinity norm of the magnetic field, we have

$$
\|\mathbf{H}\|_{L^{\infty}}^{2} \leq\|\mathbf{M}\|_{L^{\infty}}^{2}+2\|\mathbf{H}\|_{L^{2}}\|\operatorname{curl} \mathbf{H}\|_{L^{2}}
$$

Proof. First let us recall that for all $u \in H^{1}(\mathbb{R})$, we have

$$
\|u\|_{L^{\infty}}^{2} \leq 2\|u\|_{L^{2}}\left\|\frac{\partial u}{\partial x}\right\|_{L^{2}}
$$

as $\mathcal{D}(\mathbb{R})$ is dense in $H^{1}(\mathbb{R})$. Then it suffices to apply (67) to the transverse components of $\mathbf{H}$ and to recall, for the longitudinal component, that $H_{x}=-M_{x}$.

Now we can give the three steps of our proof.

- Step 1: Estimates on M.

As far as we know at this point, $\mathbf{M} \in C^{1}\left(0, T_{\max } ; H^{1}(\mathbb{R})^{3}\right)$ but it is not difficult to prove that $\mathbf{M} \in$ $C^{2}\left(0, T_{\max } ; H^{1}(\mathbb{R})^{3}\right)$, and moreover that $\|\mathbf{M}(t)\|_{L^{\infty}}=\left\|\mathbf{M}_{0}\right\|_{L^{\infty}}$. Indeed

$$
\frac{\partial \mathbf{M}}{\partial t}=\mathbf{H}_{T} \times \mathbf{M}+\frac{\alpha}{|\mathbf{M}|} \mathbf{M} \times\left(\mathbf{H}_{T} \times \mathbf{M}\right)
$$

and as we know that $\mathbf{H} \in C^{1}\left(0, T_{\max } ; L^{2}(\mathbb{R})^{3}\right)$, it is clear that

$$
\mathbf{H}_{T}=\left[\mathbf{H}+\mathbf{H}_{s}+\mathbf{H}_{a}(\mathbf{M})\right] \in C^{1}\left(0, T_{\max } ; L^{2}(\mathbb{R})^{3}\right)
$$

since $\mathbf{H}_{a}(\mathbf{M})$ linearly depends on $\mathbf{M}$. Moreover we know that

$$
\mathbf{M} \in C^{1}\left(0, T_{\max } ; H^{1}(\mathbb{R})^{3}\right) \hookrightarrow C^{1}\left(0, T_{\max } ; L^{\infty}(\mathbb{R})^{3}\right)
$$

We can conclude that $\mathbf{H}_{T} \times \mathbf{M}$ and $\mathbf{M} \times\left(\mathbf{H}_{T} \times \mathbf{M}\right)$ are in $C^{1}\left(0, T_{\max } ; L^{2}(\mathbb{R})^{3}\right)$.

Thus $\mathbf{M} \in C^{2}\left(0, T_{\max } ; L^{2}(\mathbb{R})^{3}\right)$.

- Step 2: A new energy-type identity.

The idea is to do the same kind of computations we made to get the decay in time of the electromagnetic energy but for the time derivatives of the fields $\mathbf{E}$ and $\mathbf{H}$. Let us give a formal proof assuming that $\mathbf{E}$ and $\mathbf{H}$ are smooth enough. We start from

$$
\left\{\begin{array}{l}
\frac{\partial^{2} \mathbf{H}}{\partial t^{2}}+\frac{\partial^{2} \mathbf{M}}{\partial t^{2}}=-\nabla \times \frac{\partial \mathbf{E}}{\partial t} \\
\frac{\partial^{2} \mathbf{E}}{\partial t^{2}}=\nabla \times \frac{\partial \mathbf{H}}{\partial t}
\end{array}\right.
$$

Then we multiply by $\frac{\partial \mathbf{H}}{\partial t}$ and $\frac{\partial \mathbf{E}}{\partial t}$ and integrate in space to obtain

$$
\frac{1}{2} \frac{\mathrm{d}}{\mathrm{d} t}\left[\int_{\mathbb{R}}\left(\left|\frac{\partial \mathbf{E}}{\partial t}\right|^{2}+\left|\frac{\partial \mathbf{H}}{\partial t}\right|^{2}\right) \mathrm{d} x\right]=-\int_{\mathbb{R}} \frac{\partial^{2} \mathbf{M}}{\partial t^{2}} \cdot \frac{\partial \mathbf{H}}{\partial t} \mathrm{~d} x
$$


or, since $\frac{\partial \mathbf{E}}{\partial t}=\operatorname{curl} \mathbf{H}$,

$$
\frac{1}{2} \frac{\mathrm{d}}{\mathrm{d} t}\left[\int_{\mathbb{R}}\left(|\operatorname{curl} \mathbf{H}|^{2}+\left|\frac{\partial \mathbf{H}}{\partial t}\right|^{2}\right) \mathrm{d} x\right]=-\int_{\mathbb{R}} \frac{\partial^{2} \mathbf{M}}{\partial t^{2}} \cdot \frac{\partial \mathbf{H}}{\partial t} \mathrm{~d} x .
$$

This reduces to, $\forall t \leq T_{\max }$

$$
\frac{1}{2} \int_{\mathbb{R}}\left(|\operatorname{curl} \mathbf{H}(t)|^{2}+\left|\frac{\partial \mathbf{H}}{\partial t}(t)\right|^{2}\right) \mathrm{d} x=\frac{1}{2} \int_{\mathbb{R}}\left(|\operatorname{curl} \mathbf{H}(0)|^{2}+\left|\frac{\partial \mathbf{H}}{\partial t}(0)\right|^{2}\right) \mathrm{d} x-\int_{0}^{t} \int_{\mathbb{R}} \frac{\partial^{2} \mathbf{M}}{\partial t^{2}} \cdot \frac{\partial \mathbf{H}}{\partial t} \mathrm{~d} x \mathrm{~d} s .
$$

As we know that $\forall t \in\left[0, T_{\max }\right]$

$$
\frac{\partial \mathbf{H}}{\partial t}=-\operatorname{curl} \mathbf{E}-\frac{\partial \mathbf{M}}{\partial t}=-\operatorname{curl} \mathbf{E}-\mathbf{L}_{G}^{\alpha}(\mathbf{M}, \mathbf{H})
$$

we introduce $\Lambda_{0}=-\operatorname{curl} \mathbf{E}_{0}-\mathbf{L}_{G}^{\alpha}\left(\mathbf{M}_{0}, \mathbf{H}_{0}\right) \in L^{2}$. ( $\Lambda_{0}$ is well defined since $\mathbf{E}_{0}$ and $\mathbf{H}_{0}$ are taken in $H(\operatorname{curl}, \mathbb{R})$ and $\mathbf{M}_{0}$ is taken in $H^{1}(\mathbb{R})^{3}$.) Then (72) is equivalent to

$$
\frac{1}{2}\left(\|\operatorname{curl} \mathbf{H}(t)\|_{L^{2}}^{2}+\left\|\frac{\partial \mathbf{H}}{\partial t}(t)\right\|_{L^{2}}^{2}\right)=\frac{1}{2}\left(\left\|\operatorname{curl} \mathbf{H}_{0}\right\|_{L^{2}}^{2}+\left\|\Lambda_{0}\right\|_{L^{2}}^{2}\right)-\int_{0}^{t} \int_{\mathbb{R}} \frac{\partial^{2} \mathbf{M}}{\partial t^{2}} \cdot \frac{\partial \mathbf{H}}{\partial t} \mathrm{~d} x \mathrm{~d} s .
$$

Now, to give a rigorous proof of (74), since $\mathbf{E}$ and $\mathbf{H}$ are not regular enough, we have to use a discrete differentiation. More precisely, for any Banach space $X$ and any $u \in C^{0}\left(\left[0, T^{*}[; X)\right.\right.$, we introduce

$$
\left.\forall T<T^{*}, \forall h \in\right] 0 ; T^{*}-T\left[, \quad D_{h} u(t)=\frac{u(t+h)-u(t)}{h} \in C^{0}(0, T ; X) .\right.
$$

Moreover, if $u \in C^{1}\left(\left[0, T^{*}[; X)\right.\right.$, then, as $h \rightarrow 0$,

$$
D_{h} u(t) \longrightarrow \frac{\partial u}{\partial t}(t) \quad \text { in } C^{0}(0, T ; X)
$$

Applying $D_{h}$ to Maxwell's equations - with $T^{*}=T_{\max }-$, we get by linearity

$$
\left\{\begin{array}{l}
\frac{\partial}{\partial t}\left(D_{h} \mathbf{E}\right)-\operatorname{curl}\left(D_{h} \mathbf{H}\right)=0 \\
\frac{\partial}{\partial t}\left(D_{h} \mathbf{H}\right)+\operatorname{curl}\left(D_{h} \mathbf{E}\right)=-\frac{\partial}{\partial t}\left(D_{h} \mathbf{M}\right)
\end{array} .\right.
$$

We multiply the first equation by $D_{h} \mathbf{E}$ and the second one by $D_{h} \mathbf{H}$ and then integrate the sum to get

$$
\frac{1}{2} \frac{\mathrm{d}}{\mathrm{d} t}\left(\left\|D_{h} \mathbf{E}\right\|_{L^{2}}^{2}+\left\|D_{h} \mathbf{H}\right\|_{L^{2}}^{2}\right)=-\int_{\mathbb{R}} D_{h} \frac{\partial \mathbf{M}}{\partial t} \cdot D_{h} \mathbf{H} \mathrm{d} x
$$

We now integrate in time, for any $t \in\left[0, T_{\max }[\right.$,

$$
\frac{1}{2}\left(\left\|D_{h} \mathbf{E}(t)\right\|_{L^{2}}^{2}+\left\|D_{h} \mathbf{H}(t)\right\|_{L^{2}}^{2}\right)=\frac{1}{2}\left(\left\|D_{h} \mathbf{E}(0)\right\|_{L^{2}}^{2}+\left\|D_{h} \mathbf{H}(0)\right\|_{L^{2}}^{2}\right)-\int_{0}^{t} \int_{\mathbb{R}} D_{h} \frac{\partial \mathbf{M}}{\partial t} \cdot D_{h} \mathbf{H} \mathrm{d} x \mathrm{~d} s .
$$


As $\mathbf{E} \in C^{1}\left(0, T_{\max } ; L^{2}(\mathbb{R})\right)^{3}$ and $\mathbf{H} \in C^{1}\left(0, T_{\max } ; L^{2}(\mathbb{R})\right)^{3}$, we have

$$
\left\{\begin{array}{l}
\lim _{h \rightarrow 0}\left\|D_{h} \mathbf{E}(t)\right\|_{L^{2}}^{2}=\left\|\frac{\partial \mathbf{E}}{\partial t}(t)\right\|_{L^{2}}^{2}=\|\operatorname{curl} \mathbf{H}(t)\|_{L^{2}}^{2} \\
\lim _{h \rightarrow 0}\left\|D_{h} \mathbf{H}(t)\right\|_{L^{2}}^{2}=\left\|\frac{\partial \mathbf{H}}{\partial t}(t)\right\|_{L^{2}}^{2}
\end{array} .\right.
$$

Moreover, as $\mathbf{H} \in C^{1}\left(0, T ; L^{2}(\mathbb{R})\right)^{3}$ and $\mathbf{M} \in C^{2}\left(0, T ; L^{2}(\mathbb{R})\right)^{3}$, we have, $\forall t \in\left[0, T_{\max }[\right.$,

$$
\lim _{h \rightarrow 0} \int_{0}^{t} \int_{\mathbb{R}} D_{h} \frac{\partial \mathbf{M}}{\partial t} \cdot D_{h} \mathbf{H} \mathrm{d} x \mathrm{~d} s=\int_{0}^{t} \int_{\mathbb{R}} \frac{\partial^{2} \mathbf{M}}{\partial t^{2}} \cdot \frac{\partial \mathbf{H}}{\partial t} \mathrm{~d} x \mathrm{~d} s .
$$

It is thus possible to take the limit as $h \rightarrow 0$ in (77) and then to get (74).

- Step 3: The estimate for $\left(\frac{\partial \mathbf{H}}{\partial t}\right)$ and (curlH).

To shorten the proof given below we shall consider here the case $\left(\alpha=0, \mathbf{H}_{\text {eff }}(\mathbf{M})=0\right)$ which presents all the difficulties of the case $\left(\alpha \neq 0, \mathbf{H}_{\text {eff }}(\mathbf{M}) \neq 0\right)$ that we shall detail in Appendix A. In other words we assume here that

$$
\frac{\partial \mathbf{M}}{\partial t}=\mathbf{H} \times \mathbf{M}
$$

while the case of the complete (LLG) is considered in the appendix.

In the case $\left(\alpha=0, \mathbf{H}_{\mathrm{eff}}(\mathbf{M})=0\right)$, it is easy to compute at each point:

$$
\frac{\partial^{2} \mathbf{M}}{\partial t^{2}} \cdot \frac{\partial \mathbf{H}}{\partial t}=\left[\frac{\partial \mathbf{H}}{\partial t} \times \mathbf{M}+\mathbf{H} \times \frac{\partial \mathbf{M}}{\partial t}\right] \cdot \frac{\partial \mathbf{H}}{\partial t}=-[(\mathbf{H} \times \mathbf{M}) \times \mathbf{H}] \cdot \frac{\partial \mathbf{H}}{\partial t}
$$

which reduces to

$$
\left|\left(\frac{\partial^{2} \mathbf{M}}{\partial t^{2}} \cdot \frac{\partial \mathbf{H}}{\partial t}\right)\right| \leq|\mathbf{M}||\mathbf{H}|^{2}\left|\frac{\partial \mathbf{H}}{\partial t}\right| .
$$

Thus, plugging (81) into (74), we obtain

$$
\frac{1}{2}\left(\left\|\frac{\partial \mathbf{H}}{\partial t}\right\|_{L^{2}}^{2}+\|\operatorname{curl} \mathbf{H}\|_{L^{2}}^{2}\right) \leq \frac{1}{2}\left(\left\|\operatorname{curl} \mathbf{H}_{0}\right\|_{L^{2}}^{2}+\left\|\Lambda_{0}\right\|_{L^{2}}^{2}\right)+\|\mathbf{M}\|_{L^{\infty}} \int_{0}^{t} \int_{\mathbb{R}}|\mathbf{H}|^{2}\left|\frac{\partial \mathbf{H}}{\partial t}\right| \mathrm{d} x \mathrm{~d} s .
$$

We then note that

$$
\int_{\mathbb{R}}|\mathbf{H}|^{2}\left|\frac{\partial \mathbf{H}}{\partial t}\right| \mathrm{d} x \leq\|\mathbf{H}\|_{L^{\infty}}\left\|\frac{\partial \mathbf{H}}{\partial t}\right\|_{L^{2}}\|\mathbf{H}\|_{L^{2}}
$$

that is to say, applying Lemma 5 ,

$$
\begin{aligned}
\int_{\mathbb{R}}|\mathbf{H}|^{2}\left|\frac{\partial \mathbf{H}}{\partial t}\right| \mathrm{d} x & \leq\left(\|\mathbf{M}\|_{L^{\infty}}^{2}+2\|\mathbf{H}\|_{L^{2}}\|\operatorname{curl} \mathbf{H}\|_{L^{2}}\right)^{1 / 2}\|\mathbf{H}\|_{L^{2}}\left\|\frac{\partial \mathbf{H}}{\partial t}\right\|_{L^{2}}\left\|\frac{\partial \mathbf{H}}{}\right\|_{L^{2}} .
\end{aligned}
$$


In the case $\left(\alpha=0, \mathbf{H}_{\text {eff }}(\mathbf{M})=0\right),(31)$ gives:

$$
\|\mathbf{E}\|_{L^{2}}^{2}+\|\mathbf{H}\|_{L^{2}}^{2}=\left\|\mathbf{E}_{0}\right\|_{L^{2}}^{2}+\left\|\mathbf{H}_{0}\right\|_{L^{2}}^{2} .
$$

Introducing

$$
\mathcal{E}_{0}=\frac{1}{2}\left(\left\|\mathbf{E}_{0}\right\|_{L^{2}}^{2}+\left\|\mathbf{H}_{0}\right\|_{L^{2}}^{2}\right)
$$

we get

$$
\int_{\mathbb{R}}|\mathbf{H}|^{2}\left|\frac{\partial \mathbf{H}}{\partial t}\right| \mathrm{d} x \leq \sqrt{2}\left(2 \mathcal{E}_{0}\right)^{3 / 4}\|\operatorname{curl} \mathbf{H}\|_{L^{2}}^{1 / 2}\left\|\frac{\partial \mathbf{H}}{\partial t}\right\|_{L^{2}}+\left(2 \mathcal{E}_{0}\right)^{1 / 2}\left\|\mathbf{M}_{0}\right\|_{L^{\infty}}\left\|\frac{\partial \mathbf{H}}{\partial t}\right\|_{L^{2}} .
$$

Therefore, with $C=\operatorname{Max}\left(\sqrt{2}\left(2 \mathcal{E}_{0}\right)^{3 / 4}\left\|\mathbf{M}_{0}\right\|_{L^{\infty}},\left(2 \mathcal{E}_{0}\right)^{1 / 2}\left\|\mathbf{M}_{0}\right\|_{L^{\infty}}^{2}\right)$, we get from (82) and (87)

$$
\frac{1}{2}\left(\left\|\frac{\partial \mathbf{H}}{\partial t}\right\|_{L^{2}}^{2}+\|\operatorname{curl} \mathbf{H}\|_{L^{2}}^{2}\right) \leq \frac{1}{2}\left(\left\|\operatorname{curl} \mathbf{H}_{0}\right\|_{L^{2}}^{2}+\left\|\Lambda_{0}\right\|_{L^{2}}^{2}\right)+C \int_{0}^{t}\left(1+\|\operatorname{curl} \mathbf{H}\|_{L^{2}}^{1 / 2}\right)\left\|\frac{\partial \mathbf{H}}{\partial t}\right\|_{L^{2}} \mathrm{~d} s
$$

and so

$$
\begin{aligned}
\frac{1}{2}\left(\left\|\frac{\partial \mathbf{H}}{\partial t}\right\|_{L^{2}}^{2}+\|\operatorname{curl} \mathbf{H}\|_{L^{2}}^{2}\right) & \leq \frac{1}{2}\left(\left\|\operatorname{curl} \mathbf{H}_{0}\right\|_{L^{2}}^{2}+\left\|\Lambda_{0}\right\|_{L^{2}}^{2}\right) \\
& +\frac{C}{2} \int_{0}^{t}\left(\|\operatorname{curl} \mathbf{H}\|_{L^{2}}+\left\|\frac{\partial \mathbf{H}}{\partial t}\right\|_{L^{2}}^{2}+2\left\|\frac{\partial \mathbf{H}}{\partial t}\right\|_{L^{2}}\right) \mathrm{d} s .
\end{aligned}
$$

Noticing that

$$
\|\operatorname{curl} \mathbf{H}\|_{L^{2}} \leq 1+\|\operatorname{curl} \mathbf{H}\|_{L^{2}}^{2}
$$

we have

$$
\left(\|\operatorname{curl} \mathbf{H}\|_{L^{2}}+\left\|\frac{\partial \mathbf{H}}{\partial t}\right\|_{L^{2}}^{2}+2\left\|\frac{\partial \mathbf{H}}{\partial t}\right\|_{L^{2}}\right) \leq \frac{3}{2}+2\left(\left\|\frac{\partial \mathbf{H}}{\partial t}\right\|_{L^{2}}^{2}+\|\operatorname{curl} \mathbf{H}\|_{L^{2}}\right) .
$$

Introducing the function $F(t)=\frac{1}{2}\left(\|\operatorname{curl} \mathbf{H}\|_{L^{2}}^{2}+\left\|\frac{\partial \mathbf{H}}{\partial t}\right\|_{L^{2}}^{2}\right)$, we see that (89) is equivalent to

$$
F(t) \leq F(0)+2 C \int_{0}^{t}\left(\frac{3}{4}+F(s)\right) \mathrm{d} s .
$$

We can then apply Gronwall's Lemma to the function $\left(\frac{3}{4}+F(t)\right)$ to get

$$
\left(\frac{3}{4}+F(t)\right) \leq\left(\frac{3}{4}+F(0)\right) \exp (2 C t)
$$


which yields

$$
\left(\|\operatorname{curl} \mathbf{H}\|_{L^{2}}^{2}+\left\|\frac{\partial \mathbf{H}}{\partial t}\right\|_{L^{2}}^{2}\right) \leq\left(\left\|\operatorname{curl} \mathbf{H}_{0}\right\|_{L^{2}}^{2}+\left\|\Lambda_{0}\right\|_{L^{2}}\right) \exp (2 C t)+\frac{3}{4}(\exp (2 C t)-1)
$$

which is exactly the kind of estimate that we were looking for.

\subsection{The global existence and uniqueness results}

We can conclude from estimates (33) and (93) that for all $T>0$, there exists $C_{H}(T)>0$ such that

$$
\forall t \in[0, T], \quad\|\mathbf{H}(t)\|_{H^{1}}<C_{H}(T) .
$$

Besides, we know that curlE $=-\left(\frac{\partial \mathbf{H}}{\partial t}+\frac{\partial \mathbf{M}}{\partial t}\right)$. We recall that $\frac{\partial \mathbf{M}}{\partial t} \in L^{2}(\mathbb{R})$ since $\mathbf{M} \in L^{\infty}(\mathbb{R})$ and $\mathbf{H} \in L^{2}(\mathbb{R})$. Thus (33) and (93) yield: for all $T>0$, there exists $C_{E}(T)>0$ such that

$$
\forall t \in[0, T], \quad\|\mathbf{E}(t)\|_{H^{1}}<C_{E}(T) .
$$

It remains to see that we can get the same conclusion for $\mathbf{M}$ by taking the derivative in space of the (LLG) equation:

$$
\begin{aligned}
\frac{\partial}{\partial t}\left(\frac{\partial \mathbf{M}}{\partial x}\right)= & \frac{\partial \mathbf{H}}{\partial x} \times \mathbf{M}+\frac{\partial \mathbf{H}_{\mathrm{eff}}}{\partial x} \times \mathbf{M}+\mathbf{H}_{T} \times \frac{\partial \mathbf{M}}{\partial x} \\
& +\frac{\alpha}{|\mathbf{M}|} \frac{\partial \mathbf{M}}{\partial x} \times\left[\left(\mathbf{H}+\mathbf{H}_{\mathrm{eff}}\right) \times \mathbf{M}\right]+\frac{\alpha}{|\mathbf{M}|} \mathbf{M} \times\left[\left(\frac{\partial \mathbf{H}}{\partial x}+\frac{\partial \mathbf{H}_{\mathrm{eff}}}{\partial x}\right) \times \mathbf{M}\right] \\
& +\frac{\alpha}{|\mathbf{M}|} \mathbf{M} \times\left[\left(\mathbf{H}+\mathbf{H}_{\mathrm{eff}}\right) \times \frac{\partial \mathbf{M}}{\partial x}\right]-\alpha\left(\frac{\mathbf{M}}{|\mathbf{M}|^{3}} \cdot \frac{\partial \mathbf{M}}{\partial x}\right) \mathbf{M} \times\left[\left(\mathbf{H}+\mathbf{H}_{\mathrm{eff}}\right) \times \mathbf{M}\right]
\end{aligned}
$$

Then, multiplying by $\frac{\partial \mathbf{M}}{\partial x}$ and integrating in space, we get:

$$
\begin{aligned}
\frac{1}{2} \frac{\mathrm{d}}{\mathrm{d} t}\left(\int_{\mathbb{R}}\left|\frac{\partial \mathbf{M}}{\partial x}\right|^{2} \mathrm{~d} x\right)= & \int_{\mathbb{R}}\left(\frac{\partial \mathbf{H}}{\partial x}, \mathbf{M}, \frac{\partial \mathbf{M}}{\partial x}\right) \mathrm{d} x+\int_{\mathbb{R}}\left(\frac{\partial \mathbf{H}_{\text {eff }}}{\partial x}, \mathbf{M}, \frac{\partial \mathbf{M}}{\partial x}\right) \mathrm{d} x \\
& -\int_{\mathbb{R}} \frac{\alpha}{|\mathbf{M}|}\left(\mathbf{M} \times \frac{\partial \mathbf{M}}{\partial x},\left(\frac{\partial \mathbf{H}}{\partial x}+\frac{\partial \mathbf{H}_{\text {eff }}}{\partial x}\right), \mathbf{M}\right) \mathrm{d} x \\
& -\int_{\mathbb{R}} \frac{\alpha}{|\mathbf{M}|}\left(\mathbf{M} \times \frac{\partial \mathbf{M}}{\partial x},\left(\mathbf{H}+\mathbf{H}_{\text {eff }}\right),\left(\frac{\partial \mathbf{M}}{\partial x}-\frac{\mathbf{M}}{|\mathbf{M}|^{2}} \cdot \frac{\partial \mathbf{M}}{\partial x} \mathbf{M}\right)\right) \mathrm{d} x
\end{aligned}
$$

where $(\mathbf{a}, \mathbf{b}, \mathbf{c})$ denotes the product $(\mathbf{a} \times \mathbf{b}) \cdot \mathbf{c}$. It is then easy to see that $(97)$ becomes

$$
\begin{aligned}
\frac{1}{2} \frac{\mathrm{d}}{\mathrm{d} t}\left(\left\|\frac{\partial \mathbf{M}}{\partial x}\right\|_{L^{2}}^{2}\right) \leq & \|\mathbf{M}\|_{L^{\infty}}\|\mathbf{H}\|_{H^{1}}\left\|\frac{\partial \mathbf{M}}{\partial x}\right\|_{L^{2}}+\|\mathbf{M}\|_{L^{\infty}}\left\|\mathbf{H}_{\mathrm{eff}}\right\|_{H^{1}}\left\|\frac{\partial \mathbf{M}}{\partial x}\right\|_{L^{2}} \\
& +\alpha\|\mathbf{M}\|_{L^{\infty}}\left\|\frac{\partial \mathbf{H}}{\partial x}\right\|_{L^{2}}\left\|\frac{\partial \mathbf{M}}{\partial x}\right\|_{L^{2}}+\alpha\|\mathbf{M}\|_{L^{\infty}}\left\|\frac{\partial \mathbf{H}_{\mathrm{eff}}}{\partial x}\right\|_{L^{2}}\left\|\frac{\partial \mathbf{M}}{\partial x}\right\|_{L^{2}} \\
& +2 \alpha\|\mathbf{H}\|_{L^{2}}\left\|\frac{\partial \mathbf{M}}{\partial x}\right\|_{L^{2}}^{2}+2 \alpha\left\|\mathbf{H}_{\mathrm{eff}}\right\|_{L^{2}}\left\|\frac{\partial \mathbf{M}}{\partial x}\right\|_{L^{2}}^{2} .
\end{aligned}
$$


Let us recall that we know that all the terms in the right hand side of (98), but maybe $\left\|\mathbf{H}_{\mathrm{eff}}\right\|_{H^{1}}$ and $\left\|\frac{\partial \mathbf{M}}{\partial x}\right\|_{L^{2}}$, are bounded. In what concerns $\mathbf{H}_{\text {eff }}$, we use the fact that $\mathbf{H}_{\text {eff }}(\mathbf{M})$ is affine with respect to $\mathbf{M}$. Then, using the Cauchy-Schwartz inequalities, it is straightforward to see that (98) leads to: there exists $C_{1}(T)>0$ and $C_{1}(T)>0$ such that, for all $t \leq T$,

$$
\frac{\mathrm{d}}{\mathrm{d} t}\left(\left\|\frac{\partial \mathbf{M}}{\partial x}\right\|_{L^{2}}^{2}\right) \leq C_{1}(T)+C_{2}(T)\left\|\frac{\partial \mathbf{M}}{\partial x}\right\|_{L^{2}}^{2} .
$$

We can then conclude with the Gronwall's Lemma that for all $T>0$, there exists $C_{M}(T)>0$ such that

$$
\forall t \in[0, T], \quad\|\mathbf{M}(t)\|_{H^{1}}<C_{M}(T) .
$$

Finally, we can state

Theorem 4. Given $\left(\mathbf{E}_{0}, \mathbf{H}_{0}, \mathbf{M}_{0}\right) \in H(\operatorname{curl}, \mathbb{R}) \times H(\operatorname{curl}, \mathbb{R}) \times H^{1}(\mathbb{R})^{3}$, there exists a unique global strong solution of (8) so that:

$$
\left\{\begin{array}{l}
\mathbf{E} \in C^{1}\left(\mathbb{R}_{+} ; L^{2}(\mathbb{R})^{3}\right) \cap C^{0}\left(\mathbb{R}_{+} ; H^{1}(\mathbb{R})^{2}\right) \\
\mathbf{H} \in C^{1}\left(\mathbb{R}_{+} ; L^{2}(\mathbb{R})^{3}\right) \cap C^{0}\left(\mathbb{R}_{+} ; H^{1}(\mathbb{R})^{3}\right) \\
\mathbf{M} \in C^{2}\left(\mathbb{R}_{+} ; H^{1}(\mathbb{R})^{3}\right)
\end{array}\right.
$$

\subsection{About finite velocity of propagation}

Using an energy method, it is possible to show that the velocity of propagation of electromagnetic waves in ferromagnetic media is finite. The idea of our proof consists in evaluating the electromagnetic energy outside a domain which is bounded but whose width increases with time. Note that only small changes are required to obtain the same result for arbitrary dimension of the problem.

More precisely, let us assume that there exists two reals $a$ and $b$ such that, at time $t=0$,

1. $\mathbf{E}_{0}$ and $\mathbf{H}_{0}$ are equal to 0 outside the interval $[a, b]$;

2. the initial distribution $\mathbf{M}_{0}$, outside $[a, b]$, corresponds to a stable steady state; that means that $\mathbf{M}_{0}$ is parallel to $\left(\mathbf{H}_{s}+\mathbf{H}_{a}\left(\mathbf{M}_{0}\right)\right)$ outside $[a, b]$ and that the function

$$
U(\mathbf{M})=\frac{\mu_{0}}{2}\left(K|P(\mathbf{M})|^{2}+\left|\mathbf{H}_{s}-\mathbf{M}\right|^{2}\right)
$$

reaches a global minimum, almost everywhere outside $[a, b]$, for $\mathbf{M}=\mathbf{M}_{0}$.

In this case, it is possible to multiply Maxwell's equations by $\mathbf{H}$ and $\mathbf{E}$ and to integrate the result in space as follows:

$$
\int_{b+v t}^{+\infty}\left(\varepsilon_{0} \mathbf{E} \cdot \frac{\partial \mathbf{E}}{\partial t}+\mu_{0} \mathbf{H} \cdot \frac{\partial \mathbf{H}}{\partial t}\right) \mathrm{d} x+\mu_{0} \int_{b+v t}^{+\infty} \mathbf{H} \cdot \frac{\partial \mathbf{M}}{\partial t} \mathrm{~d} x+\int_{b+v t}^{+\infty} \nabla \cdot(\mathbf{E} \times \mathbf{H}) \mathrm{d} x=0 .
$$

Our aim is to estimate the electromagnetic energy for all $x$ greater than $b+v t$, the positive constant $v$ being defined later. Equation (103) can also be formulated as

$$
\int_{b+v t}^{+\infty} \frac{\mathrm{d}}{\mathrm{d} t}\left[\frac{\varepsilon_{0}}{2}|\mathbf{E}|^{2}+\frac{\mu_{0}}{2}|\mathbf{H}|^{2}+K(x)\right] \mathrm{d} x+\mu_{0} \int_{b+v t}^{+\infty} \mathbf{H} \cdot \frac{\partial \mathbf{M}}{\partial t} \mathrm{~d} x+\int_{b+v t}^{+\infty} \nabla \cdot(\mathbf{E} \times \mathbf{H}) \mathrm{d} x=0
$$


where we denote by $(\mathbf{E} \times \mathbf{H})(x)$ the scalar function $(E y(x) H z(x)-E z(x) H y(x))$. In this expression, the function $K(x)$ must be taken constant in time. Our choice consists in $K(x)=-U\left(\mathbf{M}_{0}\right)$. Then, from (104), the computations unfold as in the general case: by adding $\mathbf{H}_{\mathrm{eff}}(\mathbf{M})$ to $\mathbf{H}$, we obtain

$$
\begin{aligned}
\int_{b+v t}^{+\infty} \frac{\mathrm{d}}{\mathrm{d} t}\left[\frac{\varepsilon_{0}}{2}|\mathbf{E}|^{2}+\frac{\mu_{0}}{2}|\mathbf{H}|^{2}-U\left(\mathbf{M}_{0}\right)\right] \mathrm{d} x-\mu_{0} \int_{b+v t}^{+\infty} \mathbf{H}_{\mathrm{eff}}(\mathbf{M}) \cdot \frac{\partial \mathbf{M}}{\partial t} \mathrm{~d} x \\
+\mu_{0} \int_{b+v t}^{+\infty} \mathbf{H}_{T}(\mathbf{H}, \mathbf{M}) \cdot \frac{\partial \mathbf{M}}{\partial t} \mathrm{~d} x-(\mathbf{E} \times \mathbf{H})(b+v t)=0 .
\end{aligned}
$$

Using (27) and (29) we obtain

$$
\int_{b+v t}^{+\infty} \frac{\mathrm{d}}{\mathrm{d} t}\left[\frac{\varepsilon_{0}}{2}|\mathbf{E}|^{2}+\frac{\mu_{0}}{2}|\mathbf{H}|^{2}+U(\mathbf{M})-U\left(\mathbf{M}_{0}\right)\right] \mathrm{d} x=-\mu_{0} \frac{\alpha}{|\gamma|} \int_{b+v t}^{+\infty} \frac{1}{|\mathbf{M}|}\left|\frac{\partial \mathbf{M}}{\partial t}\right|^{2} \mathrm{~d} x+(\mathbf{E} \times \mathbf{H})(b+v t) .
$$

We deduce that

$$
\int_{b+v t}^{+\infty} \frac{\mathrm{d}}{\mathrm{d} t}\left[\frac{\varepsilon_{0}}{2}|\mathbf{E}|^{2}+\frac{\mu_{0}}{2}|\mathbf{H}|^{2}+U(\mathbf{M})-U\left(\mathbf{M}_{0}\right)\right] \mathrm{d} x \leq-\mu_{0} \frac{\alpha}{|\gamma|} \int_{b+v t}^{+\infty} \frac{1}{|\mathbf{M}|}\left|\frac{\partial \mathbf{M}}{\partial t}\right|^{2} \mathrm{~d} x+(|\mathbf{E}||\mathbf{H}|)(b+v t) .
$$

As we know that

$$
\int_{b+v t}^{+\infty} \frac{\mathrm{d}}{\mathrm{d} t} f(s) \mathrm{d} s=\frac{\mathrm{d}}{\mathrm{d} t}\left[\int_{b+v t}^{+\infty} f(s) \mathrm{d} s\right]+v \int_{\Sigma_{t}} f(s) \mathrm{d} s
$$

we can conclude that

$$
\begin{aligned}
\frac{\mathrm{d}}{\mathrm{d} t}\left[\int _ { b + v t } ^ { + \infty } \left(\frac{\varepsilon_{0}}{2}|\mathbf{E}|^{2}+\frac{\mu_{0}}{2}|\mathbf{H}|^{2}\right.\right. & \left.\left.+U(\mathbf{M})-U\left(\mathbf{M}_{0}\right)\right) \mathrm{d} x\right] \leq-\mu_{0} \frac{\alpha}{|\gamma|} \int_{b+v t}^{+\infty} \frac{1}{|\mathbf{M}|}\left|\frac{\partial \mathbf{M}}{\partial t}\right|^{2} \mathrm{~d} x \\
- & {\left[v\left(\frac{\varepsilon_{0}}{2}|\mathbf{E}|^{2}+\frac{\mu_{0}}{2}|\mathbf{H}|^{2}+U(\mathbf{M})-U\left(\mathbf{M}_{0}\right)\right)-|\mathbf{E}||\mathbf{H}|\right](b+v t) . }
\end{aligned}
$$

Because of the choice $K(x)=-U\left(\mathbf{M}_{0}\right)$, the function of the left hand side, which is the total electromagnetic energy for $x$ greater than $b$, is always positive or equal to 0 at time $t=0$. To show that that the speed of propagation is smaller than $v$, it suffices to show that this energy can not increase in time, that is to say that the derivative in time can not be positive. Since $\left(U(\mathbf{M})-U\left(\mathbf{M}_{0}\right)(b+v t)\right) \geq 0$, it remains to choose $v$ such that

$$
\left(v \frac{\varepsilon_{0}}{2}|\mathbf{E}|^{2}+v \frac{\mu_{0}}{2}|\mathbf{H}|^{2}-|\mathbf{E}||\mathbf{H}|\right)(b+v t) \geq 0 .
$$

The reader can easily check that a possible choice is $v=c_{o}=\left(\frac{1}{\varepsilon_{o} \mu_{o}}\right)^{1 / 2}$.

Of course it would remain to do the same for $x$ smaller than $a$.

\section{THE NUMERICAL METHOD}

We present in this third part the discretization of our problem, first in space and then in time. The physical constants are not taken equal to 1 any longer.

For these numerical results we shall work in a bounded interval $[0, L]$ of $\mathbb{R}$ with Dirichlet conditions for the electric field $\mathbf{E}$. The reader will easily check that the extension to this case of the existence and uniqueness theorems of Section 2 is straightforward. We introduce the space $H^{0}($ curl, $\mathbb{R})$ :

$$
H^{0}(\operatorname{curl}, \mathbb{R})=\left\{\psi \in H(\operatorname{curl}, \mathbb{R}), \psi(0) \times \mathbf{e}_{x}=0, \psi(L) \times \mathbf{e}_{x}=0\right\} .
$$


For the "Maxwell part" of our problem, our choice consists in working from the following variational formulation [8]:

$$
\begin{cases}\left(\frac{\partial \mathbf{B}}{\partial t}, \phi\right)+(\operatorname{curl} \mathbf{E}, \phi)=0, & \forall \phi \in L^{2} \\ \varepsilon_{0}\left(\frac{\partial \mathbf{E}}{\partial t}, \psi\right)-(\mathbf{H}, \operatorname{curl} \psi)=0, & \forall \psi \in H^{1} . \\ (\mathbf{H}, \phi)=\left(\frac{\mathbf{B}}{\mu_{0}}, \phi\right)-(\mathbf{M}, \phi), & \forall \phi \in L^{2}\end{cases}
$$

It is important to see that the curl term in the second equation has been integrated by parts. For this reason functions $\phi$ can be taken in $L^{2}$ rather than in $H$ (curl). The third equation looks like a triviality yet it indicates that it would be better to look for $\mathbf{H}, \mathbf{B}$ and $\mathbf{M}$ in the same functional space.

\subsection{Semi-discretization in space}

\subsubsection{Construction of the approximate}

We assume that $L=N h$, where $h>0$ is our space step:

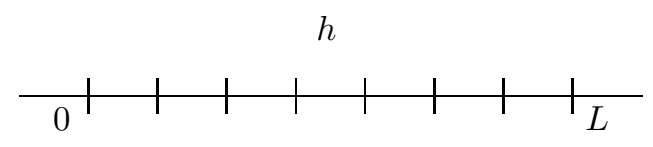

We take the discrete electric field in the space $V_{h}$ of piecewise linear functions and the discrete fields $\mathbf{H}_{h}, \mathbf{B}_{h}$ and $\mathbf{M}_{h}$ in the space $X_{h}$ of piecewise constant functions. We introduce $\left\{\psi_{i} \in P_{1}\right\}_{i=1}^{N-1}$ the canonical basis functions of $V_{h}$ to define $\mathbf{E}_{h}$ :

$$
\mathbf{E}_{h}=\sum_{i=1}^{N-1}\left(E_{y}(i) \psi_{i}(x) \mathbf{e}_{y}+E_{z}(i) \psi_{i}(x) \mathbf{e}_{z}\right)
$$

and $\left\{\phi_{i} \in P_{0}\right\}_{i=1}^{N}$ the canonical basis functions of $X_{h}$ to define $\mathbf{H}_{h}, \mathbf{B}_{h}$ and $\mathbf{M}_{h}$ :

$$
\begin{aligned}
\mathbf{B}_{h} & =\sum_{i=1}^{N}\left(B_{y}\left(i-\frac{1}{2}\right) \phi_{i}(x) \mathbf{e}_{y}+B_{z}\left(i-\frac{1}{2}\right) \phi_{i}(x) \mathbf{e}_{z}\right) \\
\mathbf{H}_{h} & =\sum_{i=1}^{N}\left(-M_{x}\left(i-\frac{1}{2}\right) \phi_{i}(x) \mathbf{e}_{x}+H_{y}\left(i-\frac{1}{2}\right) \phi_{i}(x) \mathbf{e}_{y}+H_{z}\left(i-\frac{1}{2}\right) \phi_{i}(x) \mathbf{e}_{z}\right) \\
\mathbf{M}_{h} & =\sum_{i=1}^{N}\left(M_{x}\left(i-\frac{1}{2}\right) \phi_{i}(x) \mathbf{e}_{x}+M_{y}\left(i-\frac{1}{2}\right) \phi_{i}(x) \mathbf{e}_{y}+M_{z}\left(i-\frac{1}{2}\right) \phi_{i}(x) \mathbf{e}_{z}\right) .
\end{aligned}
$$

It is now possible to write a discrete variational formulation of (112):

$$
\left\{\begin{array}{ll}
\left(\frac{\partial \mathbf{B}_{h}}{\partial t}, \phi_{h}\right)+\left(\operatorname{curl} \mathbf{E}_{h}, \phi_{h}\right)=0, & \forall \phi_{h} \in\left(P_{0}\right)^{2} \\
\varepsilon_{0}\left(\frac{\partial \mathbf{E}_{h}}{\partial t}, \psi_{h}\right)-\left(\mathbf{H}_{h}, \operatorname{curl} \psi_{h}\right)=0, & \forall \psi_{h} \in\left(P_{1}\right)^{2}
\end{array} .\right.
$$


This leads to the following scalar system for Maxwell's equations:

$$
1 \leq i \leq N, \quad\left\{\begin{array}{l}
\frac{\mathrm{d} B_{y}\left(i-\frac{1}{2}\right)}{\mathrm{d} t}=\frac{E_{z}(i)-E_{z}(i-1)}{\Delta x} \\
\frac{\mathrm{d} B_{z}\left(i-\frac{1}{2}\right)}{\mathrm{d} t}=-\frac{E_{y}(i)-E_{y}(i-1)}{\Delta x}
\end{array}\right.
$$

(where $E_{y}(0)=0=E_{z}(0)$ and $\left.E_{y}(N)=0=E_{z}(N)\right)$, and

$$
1 \leq i \leq N-1, \quad\left\{\begin{array}{rl}
\frac{\mathrm{d} E_{y}(i)}{\mathrm{d} t} & =-\frac{H_{z}\left(i+\frac{1}{2}\right)-H_{z}\left(i-\frac{1}{2}\right)}{\Delta x} \\
\frac{\mathrm{d} E_{z}(i)}{\mathrm{d} t} & =\frac{H_{y}\left(i+\frac{1}{2}\right)-H_{y}\left(i-\frac{1}{2}\right)}{\Delta x}
\end{array} .\right.
$$

With this approach and equations (119), a discrete curl operator is defined for the space $X_{h}$ (which is a space of discontinuous functions); in the 1D case it is exactly the usual operator corresponding to Yee's scheme [9], but this would be no longer true in the 2D case (see [16]). This discrete curl operator is defined with centered finite difference which guarantees order 2 in space for our scheme.

The situation is of course different for the (LLG) equation and the magnetic coupling since no derivative occurs in these equations. We simply have [see (14)]:

$$
\frac{\partial \mathbf{M}_{h}}{\partial t}=|\gamma| \mathbf{H}_{T h}(\mathbf{M}) \times \mathbf{M}_{h}+\frac{\alpha}{|\mathbf{M}|} \mathbf{M}_{h} \times \frac{\partial \mathbf{M}_{h}}{\partial t}
$$

with $\mathbf{H}_{T h}(\mathbf{M})=\mathbf{H}_{h}+\mathbf{H}_{s}-K P\left(\mathbf{M}_{h}\right)$ and $\mathbf{H}_{h}=\frac{\mathbf{B}_{h}}{\mu_{0}}-\mathbf{M}_{h}$.

\subsubsection{Existence of solutions and stability analysis}

It can be seen that the problem defined from (117) and (120) consists in solving the following system:

$$
\left\{\begin{array}{l}
\frac{\partial \mathbf{B}_{h}}{\partial t}+\operatorname{curl}_{h} \mathbf{E}_{h}=0 \\
\mathbf{B}_{h}=\mu_{0}\left(\mathbf{H}_{h}+\mathbf{M}_{h}\right) \\
\varepsilon_{0} \frac{\partial \mathbf{E}_{h}}{\partial t}-\operatorname{curl}_{h} \mathbf{H}_{h}=0 \\
\frac{\partial \mathbf{M}_{h}}{\partial t}=\mathbf{L}_{G}^{\alpha}\left(\mathbf{M}_{h}, \mathbf{H}_{h}\right)
\end{array}\right.
$$

where $\operatorname{curl}_{h}$ is the discrete curl operator defined on $\left(P_{0}\right)^{2}$ by:

$$
\forall \phi_{h} \in\left(P_{0}\right)^{2}, \forall \psi_{h} \in\left(P_{1}\right)^{2},\left(\operatorname{curl}_{h} \phi_{h}, \psi_{h}\right)=\left(\phi_{h}, \operatorname{curl} \psi_{h}\right) .
$$

We see that $(121)$ is a system of $(2+2+3) N$ ordinary differential equations. Thus the Cauchy-Lipschitz theorem applies and ensures that there exists a unique local solution $\left(\mathbf{E}_{h}, \mathbf{H}_{h}, \mathbf{M}_{h}\right)$.

Concerning this local solution, we see first that there is nothing new to say about the conservation at each point of the norm of $\mathbf{M}_{h}$, since the (LLG) equation is exactly the same as in the continuous case:

$$
\left|\mathbf{M}_{h}(x, t)\right|^{2}=\left|\mathbf{M}_{0 h}(x)\right|^{2}, \forall x \in[0, L] .
$$


To get the complete control of the local solution $\left(\mathbf{E}_{h}, \mathbf{H}_{h}, \mathbf{M}_{h}\right)$, it suffices then to prove again the decay in time of the electromagnetic energy. If we take $\phi_{h}=\mathbf{H}_{h}$ and $\psi_{h}=\mathbf{E}_{h}$ in (117) and then integrate, we obtain by adding

$$
\int_{0}^{L}\left\{\mu_{0}\left(\frac{\partial \mathbf{H}_{h}}{\partial t}, \mathbf{H}_{h}\right)+\varepsilon_{0}\left(\frac{\partial \mathbf{E}_{h}}{\partial t}, \mathbf{E}_{h}\right)\right\} \mathrm{d} x=\mu_{0} \int_{0}^{L}\left(\mathbf{H}_{h} \cdot \frac{\partial \mathbf{M}_{h}}{\partial t}\right) \mathrm{d} x
$$

We do the same computations but with the 1D assumptions:

$$
-\mu_{0} \int_{0}^{L}\left(\mathbf{H}_{a h} \cdot \frac{\partial \mathbf{M}_{h}}{\partial t}\right) \mathrm{d} x=\mu_{0} \int_{0}^{L}\left(K P\left(\mathbf{M}_{h}\right) \cdot \frac{\partial P\left(\mathbf{M}_{h}\right)}{\partial t}\right) \mathrm{d} x=\frac{1}{2} \frac{\mathrm{d}}{\mathrm{d} t}\left[\mathcal{E}_{a}\left(\mathbf{M}_{h}\right)\right] .
$$

Since the (LLG) equation has not been modified, the other results are unchanged and lead to

$$
\frac{\mathrm{d}}{\mathrm{d} t}\left[\frac{\varepsilon_{0}}{2}\left\|\mathbf{E}_{h}\right\|_{L^{2}}^{2}+\frac{\mu_{0}}{2}\left\|\mathbf{H}_{h}\right\|_{L^{2}}^{2}+\frac{\mu_{0} K}{2}\left\|P\left(\mathbf{M}_{h}\right)\right\|_{L^{2}}^{2}+\frac{\mu_{0}}{2}\left\|\mathbf{H}_{s}-\mathbf{M}_{h}\right\|_{L^{2}}^{2}\right]=-\mu_{0} \frac{\alpha}{|\gamma|} \int_{0}^{L} \frac{1}{\left|\mathbf{M}_{h}\right|}\left|\frac{\partial \mathbf{M}_{h}}{\partial t}\right|^{2} \mathrm{~d} x
$$

which is the semi-discrete equivalent of (31). Thus, as in the continuous case, we conclude that

$$
\begin{aligned}
& \varepsilon_{0}\left\|\mathbf{E}_{h}(t)\right\|_{L^{2}}^{2} \leq\left(\varepsilon_{0}\left\|\mathbf{E}_{h 0}\right\|_{L^{2}}^{2}+\mu_{0}\left\|\mathbf{H}_{h 0}\right\|_{L^{2}}^{2}+(1+K) \mu_{0}\left\|\mathbf{M}_{h 0}\right\|_{L^{2}}^{2}+\mu_{0}\left\|\mathbf{H}_{s}\right\|_{L^{2}}^{2}\right) \\
& \mu_{0}\left\|\mathbf{H}_{h}(t)\right\|_{L^{2}}^{2} \leq\left(\varepsilon_{0}\left\|\mathbf{E}_{h 0}\right\|_{L^{2}}^{2}+\mu_{0}\left\|\mathbf{H}_{h 0}\right\|_{L^{2}}^{2}+(1+K) \mu_{0}\left\|\mathbf{M}_{h 0}\right\|_{L^{2}}^{2}+\mu_{0}\left\|\mathbf{H}_{s}\right\|_{L^{2}}^{2}\right)
\end{aligned}
$$

These estimations ensure that the local solution is indeed a global solution. Moreover, they do not depend on $h$.

\subsection{Time stepping}

Our semi-discrete equations must be now discretized in time.

\subsubsection{Construction of the approximate}

We shall use to discretize (118) and (119) in time the standard leapfrog technique: if $\Delta t>0$ is our time step, the electric field is discretized at times $t^{n}=n \Delta t$ :

$$
\mathbf{E}_{h}^{n} \simeq \mathbf{E}_{h}(t=n \Delta t)
$$

while the magnetic field, the magnetic induction and the magnetization are discretized at time $t^{n+\frac{1}{2}}=\left(n+\frac{1}{2}\right) \Delta t$ :

$$
\mathbf{B}_{h}^{n+\frac{1}{2}} \simeq \mathbf{B}_{h}\left(\left(n+\frac{1}{2}\right) \Delta t\right), \mathbf{M}_{h}^{n+\frac{1}{2}} \simeq \mathbf{M}_{h}\left(\left(n+\frac{1}{2}\right) \Delta t\right), \mathbf{H}_{h}^{n+\frac{1}{2}} \simeq \mathbf{H}_{h}\left(\left(n+\frac{1}{2}\right) \Delta t\right) .
$$

Besides we denote by $\mathbf{M}_{h}^{n}$ (resp. $\mathbf{H}_{h}^{n}$ and $\mathbf{B}_{h}^{n}$ ) the half sum of $\mathbf{M}_{h}^{n-\frac{1}{2}}$ and $\mathbf{M}_{h}^{n+\frac{1}{2}}$ (resp. of $\mathbf{H}_{h}^{n-\frac{1}{2}}$ and $\mathbf{H}_{h}^{n+\frac{1}{2}}$, and of $\mathbf{B}_{h}^{n-\frac{1}{2}}$ and $\mathbf{B}_{h}^{n+\frac{1}{2}}$ ).

Discretizing the system (121), we get the following scheme: on the one hand, for Maxwell's equations,

$$
\frac{\mathbf{B}_{h}^{n+\frac{1}{2}}-\mathbf{B}_{h}^{n-\frac{1}{2}}}{\Delta t}=-\operatorname{curl}_{h} \mathbf{E}_{h}^{n}
$$


and

$$
\varepsilon_{0} \frac{\mathbf{E}_{h}^{n+1}-\mathbf{E}_{h}^{n}}{\Delta t}=\operatorname{curl}_{h} \mathbf{H}_{h}^{n+\frac{1}{2}}, \text { with } \mathbf{H}_{h}^{n+\frac{1}{2}}=\frac{\mathbf{B}_{h}^{n+\frac{1}{2}}}{\mu_{0}}-\mathbf{M}_{h}^{n+\frac{1}{2}}
$$

and on the other hand, for the (LLG) equation,

$$
\frac{\mathbf{M}_{h}^{n+\frac{1}{2}}-\mathbf{M}_{h}^{n-\frac{1}{2}}}{\Delta t}=|\gamma| \mathbf{H}_{T h}^{n} \times \mathbf{M}_{h}^{n}+\frac{\alpha}{\left|\mathbf{M}_{h}^{n}\right|} \mathbf{M}_{h}^{n} \times \frac{\mathbf{M}_{h}^{n+\frac{1}{2}}-\mathbf{M}_{h}^{n-\frac{1}{2}}}{\Delta t}
$$

with $\mathbf{H}_{T h}^{n}=\frac{1}{\mu_{0}} \mathbf{B}_{h}^{n}+\mathbf{H}_{s}-K P\left(\mathbf{M}_{h}^{n}\right)$ and $\left|\mathbf{M}_{h}^{n}\right|=\frac{\left|\mathbf{M}_{h}^{n-\frac{1}{2}}\right|+\left|\mathbf{M}_{h}^{n+\frac{1}{2}}\right|}{2}$.

When $\mathbf{B}_{h}^{n-\frac{1}{2}}, \mathbf{M}_{h}^{n-\frac{1}{2}}$ and $\mathbf{E}_{h}^{n}$ are assumed to be known, the computations at the following step unfold like this:

1. $\mathbf{B}_{h}^{n+\frac{1}{2}}$ is calculated by (130);

2. $\mathbf{M}_{h}^{n+\frac{1}{2}}$ is calculated by (132), hence $\mathbf{H}_{T h}^{n}$ can be computed;

3. $\mathbf{E}_{h}^{n+1}$ is calculated by (131).

Moreover it can be seen that our time stepping is centered which ensures an approximation of order 2 in time.

\subsubsection{Existence and actual computation of the discrete solution}

The calculation of $\mathbf{B}_{h}^{n+\frac{1}{2}}, \mathbf{H}_{h}^{n+\frac{1}{2}}$ and $\mathbf{E}_{h}^{n+1}$ from (130) and (131) is obvious but we have to make precise the way we determinate $\mathbf{M}_{h}^{n+\frac{1}{2}}$ from (132). Moreover, because of the non-linearity of this equation, we can't be sure a priori either of existence of a solution, or of uniqueness. Also we shall see that although the scheme is implicit, the computation of $\mathbf{M}_{h}^{n+1}$ can be carried out explicitly.

\section{- A new form of the discrete (LLG) equation}

First of all, we can "decrease" the non-linearity of (132) with the help of three simple mathematical remarks:

1. The conservation of the norm of the magnetization: one only has to take the scalar product of (132) by $\mathbf{M}_{h}^{n}$ to find

$$
\left(\frac{\mathbf{M}_{h}^{n+\frac{1}{2}}-\mathbf{M}_{h}^{n-\frac{1}{2}}}{\Delta t}\right) \cdot\left(\frac{\mathbf{M}_{h}^{n+\frac{1}{2}}+\mathbf{M}_{h}^{n-\frac{1}{2}}}{2}\right)=\frac{1}{2 \Delta t}\left(\left|\mathbf{M}_{h}^{n+\frac{1}{2}}\right|^{2}-\left|\mathbf{M}_{h}^{n-\frac{1}{2}}\right|^{2}\right)=0 .
$$

This means that $\left|\mathbf{M}_{h}^{n}\right|$ is a constant in (132) and the superscript $n$ can be omitted.

2. A change in the second vector product: we have

$$
\frac{\mathbf{M}_{h}^{n+\frac{1}{2}}+\mathbf{M}_{h}^{n-\frac{1}{2}}}{2} \times \frac{\mathbf{M}_{h}^{n+\frac{1}{2}}-\mathbf{M}_{h}^{n-\frac{1}{2}}}{\Delta t}=\frac{\mathbf{M}_{h}^{n+\frac{1}{2}}+\mathbf{M}_{h}^{n-\frac{1}{2}}}{2} \times \frac{\left(\mathbf{M}_{h}^{n+\frac{1}{2}}+\mathbf{M}_{h}^{n-\frac{1}{2}}\right)-2 \mathbf{M}_{h}^{n-\frac{1}{2}}}{\Delta t} .
$$

Then, (132) becomes

$$
\mathbf{M}_{h}^{n+\frac{1}{2}}-\mathbf{M}_{h}^{n-\frac{1}{2}}=\left[\frac{|\gamma| \Delta t}{2} \mathbf{H}_{T h}^{n}+\frac{\alpha}{\left|\mathbf{M}_{h}^{n}\right|} \mathbf{M}_{h}^{n-\frac{1}{2}}\right] \times\left(\mathbf{M}_{h}^{n+\frac{1}{2}}+\mathbf{M}_{h}^{n-\frac{1}{2}}\right) .
$$


3. The remark (14) about $\mathbf{H}_{a}(\mathbf{M})$ : we have

$$
-K P\left(\mathbf{M}_{h}^{n}\right) \times \mathbf{M}_{h}^{n}=K\left(\mathbf{p} \cdot \mathbf{M}_{h}^{n}\right) \mathbf{p} \times \mathbf{M}_{h}^{n} .
$$

Finally we have shown that the (LLG) equation is equivalent to

$$
\mathbf{M}_{h}^{n+\frac{1}{2}}-\mathbf{M}_{h}^{n-\frac{1}{2}}=\left[\frac{|\gamma| \Delta t}{2}\left(\frac{\mathbf{B}_{h}^{n}}{\mu_{0}}+\mathbf{H}_{s}\right)+\alpha \mathbf{M}_{h}^{n-\frac{1}{2}}+\frac{K|\gamma| \Delta t}{2}\left(\mathbf{p} \cdot \mathbf{M}_{h}^{n}\right) \mathbf{p}\right] \times\left(2 \mathbf{M}_{h}^{n}\right) .
$$

Introducing the following notations:

- $\mathbf{f}=2 \mathbf{M}_{h}^{n-\frac{1}{2}}, \quad \mathbf{a}=-\left(\frac{|\gamma| \Delta t}{2}\left(\frac{\mathbf{B}_{h}^{n}}{\mu_{0}}+\mathbf{H}_{s}\right)+\alpha \mathbf{M}_{h}^{n-\frac{1}{2}}\right)$ and $\lambda=-\frac{K|\gamma| \Delta t}{4} ;$

- $\mathbf{x}=\mathbf{M}_{h}^{n+\frac{1}{2}}+\mathbf{M}_{h}^{n-\frac{1}{2}}=2 \mathbf{M}_{h}^{n}$

we get the following non-linear vectorial equation:

$$
\mathbf{x}+\mathbf{a} \times \mathbf{x}+\lambda(\mathbf{p} \cdot \mathbf{x}) \mathbf{p} \times \mathbf{x}=\mathbf{f}
$$

where $\mathbf{f}, \mathbf{a}$ and $\lambda$ are data and $\mathbf{x}$ is our new unknown.

Remark 5. If $K$ were equal to 0 , we would get $\mathbf{x}+\mathbf{a} \times \mathbf{x}=\mathbf{f}$; in this case, Lemma 1 applies, and we conclude that

$$
\mathbf{x}=\frac{\mathbf{f}+(\mathbf{a} \cdot \mathbf{f}) \mathbf{a}-\mathbf{a} \times \mathbf{f}}{1+|\mathbf{a}|^{2}}
$$

\section{- Existence and uniqueness of the solution}

We first consider the linear function of Lemma 1

$$
A(\mathbf{x})=\mathbf{x}+\mathbf{a} \times \mathbf{x}=\mathbf{y} \Leftrightarrow x=A^{-1} \mathbf{y} .
$$

We recall that $A^{-1}$ is a contraction. Let $\mathbf{x}$ be now a solution to (138); we introduce

$$
\mathbf{x}=A^{-1}(\mathbf{f}-\lambda(\mathbf{p} \cdot \mathbf{x}) \mathbf{p} \times \mathbf{x})=F(\mathbf{x}) .
$$

It is not difficult to see that $F: B(0,1+|\mathbf{f}|) \mapsto B(0,1+|\mathbf{f}|)$ as soon as $|\lambda| \leq \frac{1}{(1+|\mathbf{f}|)^{2}}$ :

$$
|F(\mathbf{x})| \leq|\mathbf{f}|+|\lambda||\mathbf{x}|^{2} \leq|\mathbf{f}|+|\lambda|(1+|\mathbf{f}|)^{2} .
$$

Then we compute

$$
\begin{aligned}
|F(\mathbf{x})-F(\mathbf{y})| & \leq|\lambda||(\mathbf{p} \cdot \mathbf{x}) \mathbf{p} \times \mathbf{x}-(\mathbf{p} \cdot \mathbf{y}) \mathbf{p} \times \mathbf{y}| \\
& \leq|\lambda||(\mathbf{p} \cdot \mathbf{x})(\mathbf{p} \times \mathbf{x}-\mathbf{p} \times \mathbf{y})-(\mathbf{p} \cdot \mathbf{x}-\mathbf{p} \cdot \mathbf{y}) \mathbf{p} \times \mathbf{y}| \\
& \leq|\lambda||(\mathbf{p} \cdot \mathbf{x}) \mathbf{p} \times(\mathbf{x}-\mathbf{y})-\mathbf{p} \cdot(\mathbf{x}-\mathbf{y}) \mathbf{p} \times \mathbf{y}| .
\end{aligned}
$$

Hence

$$
|F(\mathbf{x})-F(\mathbf{y})| \leq 2|\lambda|(1+|\mathbf{f}|)|\mathbf{x}-\mathbf{y}|
$$


We conclude that $F$ is a contraction from $B(0,1+|\mathbf{f}|)$ to $B(0,1+|\mathbf{f}|)$ as soon as

$$
|\lambda| \leq \operatorname{Min}\left([2|\lambda|(1+|\mathbf{f}|)]^{-1},(1+|\mathbf{f}|)^{-2}\right) .
$$

Therefore, for $\Delta t$ small enough, the contraction theorem applies and there exists a unique solution to (138).

\section{- Actual computation}

If we tried to expand (138), a quadratic vectorial equation, it would lead to a scalar polynomial of degree 6 . We should use in this case some iterative method and give up the hope of an explicit scheme.

To avoid this difficulty, our idea consists in projecting - when it is possible - the vectorial unknown $\mathbf{x}$ in the basis $(\mathbf{a}, \mathbf{p}, \mathbf{a} \times \mathbf{p})$. We distinguish two cases

1. $|\mathbf{a} \times \mathbf{p}|=0$.

Then $(\mathbf{a}, \mathbf{p}, \mathbf{a} \times \mathbf{p})$ is not a basis, but there exits $\theta \in \mathbb{R}$ such that $\mathbf{a}=\theta \mathbf{p}$. Thus equation (138) is equivalent to

$$
\mathbf{x}+[\theta+\lambda(\mathbf{p} \cdot \mathbf{x})] \mathbf{p} \times \mathbf{x}=\mathbf{f} .
$$

Taking the scalar product by $\mathbf{p}$ yields $(\mathbf{p} \cdot \mathbf{x})=(\mathbf{p} \cdot \mathbf{f})$, so that $(138)$ is equivalent to

$$
\mathbf{x}+[\theta+\lambda(\mathbf{p} \cdot \mathbf{f})] \mathbf{p} \times \mathbf{x}=\mathbf{f}
$$

and Lemma 1 applies. The solution is explicit.

2. $|\mathbf{a} \times \mathbf{p}| \neq 0$.

We can work in the basis $(\mathbf{a}, \mathbf{p}, \mathbf{a} \times \mathbf{p})$ and get a system of three equations in scalar unknowns $X=(\mathbf{a} \cdot \mathbf{x})$, $Y=(\mathbf{p} \cdot \mathbf{x})$ and $Z=(\mathbf{a} \times \mathbf{p}) \cdot \mathbf{x}$ by taking the scalar product of (138) by $\mathbf{a}, \mathbf{p}$ and $\mathbf{a} \times \mathbf{p}$ :

$$
\left\{\begin{array}{l}
Y-Z=(\mathbf{p} \cdot \mathbf{f}) \\
X+\lambda Y Z=(\mathbf{a} \cdot \mathbf{f}) \\
Z-(\mathbf{a} \cdot \mathbf{p}) X+\|\mathbf{a}\|^{2} Y+\lambda(\mathbf{a} \cdot \mathbf{p}) Y^{2}-\lambda X Y=(\mathbf{a} \times \mathbf{p}) \cdot \mathbf{f}
\end{array} .\right.
$$

This leads to

$$
\left\{\begin{array}{l}
Y=Z+(\mathbf{p} \cdot \mathbf{f}) \\
X=(\mathbf{a} \cdot \mathbf{f})-\lambda Z(Z+(\mathbf{p} \cdot \mathbf{f})) \\
\lambda^{2} Z^{3}+2 \lambda((\mathbf{a} \cdot \mathbf{p})+\lambda(\mathbf{p} \cdot \mathbf{f})) Z^{2}+\left(1+\|\mathbf{a}\|^{2}-\lambda(\mathbf{a} \cdot \mathbf{f})+3 \lambda(\mathbf{a} \cdot \mathbf{p})(\mathbf{p} \cdot \mathbf{f})+\lambda^{2}(\mathbf{p} \cdot \mathbf{f})^{2}\right) Z \\
\quad-\lambda(\mathbf{a} \cdot \mathbf{f})(\mathbf{p} \cdot \mathbf{f})-(\mathbf{a} \cdot \mathbf{p})(\mathbf{p} \cdot \mathbf{f})+\lambda(\mathbf{a} \cdot \mathbf{p})(\mathbf{p} \cdot \mathbf{f})^{2}+\|\mathbf{a}\|^{2}(\mathbf{p} \cdot \mathbf{f})-(\mathbf{a} \times \mathbf{p}) \cdot \mathbf{f}=0 .
\end{array}\right.
$$

Solving this system is equivalent to finding the - unique - real root of a polynomial of degree 3 in $Z$, while degree 6 was expected. This time again, solution is given explicitly. Knowing $X, Y$ and $Z$, we can determinate $\mathrm{x}$ by:

$$
\mathbf{x}=\frac{1}{|\mathbf{a} \times \mathbf{p}|^{2}}\left[(X-(\mathbf{a} \cdot \mathbf{p}) Y) \mathbf{a}+\left(|\mathbf{a}|^{2} Y-(\mathbf{a} \cdot \mathbf{p}) X\right) \mathbf{p}+Z \mathbf{a} \times \mathbf{p}\right]
$$




\subsubsection{Stability analysis}

We want to show the decay at each time step of a discrete electromagnetic energy by adapting the proof of the continuous case. Multiplying (130) by $\Delta t\left(\mathbf{H}_{h}^{n+\frac{1}{2}}+\mathbf{H}_{h}^{n-\frac{1}{2}}\right)$, we obtain

$$
\begin{array}{r}
\mu_{0}\left\|\mathbf{H}_{h}^{n+\frac{1}{2}}\right\|^{2}-\mu_{0}\left\|\mathbf{H}_{h}^{n-\frac{1}{2}}\right\|^{2}+\mu_{0}\left(\mathbf{M}_{h}^{n+\frac{1}{2}}-\mathbf{M}_{h}^{n-\frac{1}{2}}, \frac{\mathbf{B}_{h}^{n+\frac{1}{2}}+\mathbf{B}_{h}^{n-\frac{1}{2}}}{\mu_{0}}-\left(\mathbf{M}_{h}^{n+\frac{1}{2}}+\mathbf{M}_{h}^{n-\frac{1}{2}}\right)\right) \\
+\Delta t\left(\operatorname{curl}_{h} \mathbf{E}_{h}^{n}, \mathbf{H}_{h}^{n+\frac{1}{2}}+\mathbf{H}_{h}^{n-\frac{1}{2}}\right)=0
\end{array}
$$

Using the conservation of $\left|\mathbf{M}_{h}\right|$, the fact that the operator $\operatorname{curl}_{h}$ as we defined it is a self-adjoint operator, and (131), we see that (151) is equivalent to

$$
\left[\mu_{0}\left\|\mathbf{H}_{h}^{n+\frac{1}{2}}\right\|^{2}+\varepsilon_{0}\left(\mathbf{E}_{h}^{n}, \mathbf{E}_{h}^{n+1}\right)\right]-\left[\mu_{0}\left\|\mathbf{H}_{h}^{n-\frac{1}{2}}\right\|^{2}+\varepsilon_{0}\left(\mathbf{E}_{h}^{n-1}, \mathbf{E}_{h}^{n}\right)\right]=-\mu_{0}\left(\mathbf{M}_{h}^{n+\frac{1}{2}}-\mathbf{M}_{h}^{n-\frac{1}{2}}, \frac{2 \mathbf{B}_{h}^{n}}{\mu_{0}}\right) .
$$

By adding $2 \mathbf{H}_{\text {eff }}\left(\mathbf{M}_{h}^{n}\right)$ to $\frac{2 \mathbf{B}_{h}^{n}}{\mu_{0}}$, we obtain

$$
\begin{aligned}
{\left[\mu_{0}\left\|\mathbf{H}_{h}^{n+\frac{1}{2}}\right\|^{2}+\varepsilon_{0}\left(\mathbf{E}_{h}^{n}, \mathbf{E}_{h}^{n+1}\right)\right]-\left[\mu_{0}\left\|\mathbf{H}_{h}^{n-\frac{1}{2}}\right\|^{2}+\varepsilon_{0}\left(\mathbf{E}_{h}^{n-1}, \mathbf{E}_{h}^{n}\right)\right] } \\
-\mu_{0}\left(\mathbf{M}_{h}^{n+\frac{1}{2}}-\mathbf{M}_{h}^{n-\frac{1}{2}}, 2 \mathbf{H}_{\mathrm{eff}}\left(\mathbf{M}_{h}^{n}\right)\right)=-\mu_{0}\left(\mathbf{M}_{h}^{n+\frac{1}{2}}-\mathbf{M}_{h}^{n-\frac{1}{2}}, 2 \mathbf{H}_{T h}^{n}\right) .
\end{aligned}
$$

Let us compute the left hand side; we have

$$
\mu_{0}\left(2 K P\left(\mathbf{M}_{h}^{n}\right), \mathbf{M}_{h}^{n+\frac{1}{2}}-\mathbf{M}_{h}^{n-\frac{1}{2}}\right)=2 K \mu_{0}\left|P\left(\mathbf{M}_{h}^{n+\frac{1}{2}}\right)\right|^{2}-2 K \mu_{0}\left|P\left(\mathbf{M}_{h}^{n-\frac{1}{2}}\right)\right|^{2}
$$

Finally we obtain that $-\mu_{0}\left(\mathbf{M}_{h}^{n+\frac{1}{2}}-\mathbf{M}_{h}^{n-\frac{1}{2}}, 2 \mathbf{H}_{\text {eff }}\left(\mathbf{M}_{h}^{n}\right)\right)$ is equal to

$$
\left[2 K \mu_{0}\left|P\left(\mathbf{M}_{h}^{n+\frac{1}{2}}\right)\right|^{2}-2 \mu_{0} \mathbf{H}_{s} \cdot \mathbf{M}_{h}^{n+\frac{1}{2}}\right]-\left[2 K \mu_{0}\left|P\left(\mathbf{M}_{h}^{n-\frac{1}{2}}\right)\right|^{2}-2 \mu_{0} \mathbf{H}_{s} \cdot \mathbf{M}_{h}^{n-\frac{1}{2}}\right] \text {. }
$$

Further one has only to take the vector product of (132) by $\mathbf{M}_{h}^{n+\frac{1}{2}}-\mathbf{M}_{h}^{n-\frac{1}{2}}$ to get

$$
-\mu_{0}\left(\mathbf{M}_{h}^{n+\frac{1}{2}}-\mathbf{M}_{h}^{n-\frac{1}{2}}, \mathbf{H}_{T h}^{n}\right)=-\frac{\alpha}{|\gamma|} \mu_{0}\left|\mathbf{M}_{h}^{n+\frac{1}{2}}-\mathbf{M}_{h}^{n-\frac{1}{2}}\right|^{2}
$$

Thus, if we introduce, $\forall n \in \mathbb{N}$,

$$
U_{h}\left(n+\frac{1}{2}\right)=\mu_{0}\left|\mathbf{H}_{h}^{n+\frac{1}{2}}\right|^{2}+\varepsilon_{0}\left(\mathbf{E}_{h}^{n}, \mathbf{E}_{h}^{n+1}\right)+2 K \mu_{0}\left|P\left(\mathbf{M}_{h}^{n+\frac{1}{2}}\right)\right|^{2}+\mu_{0}\left|\mathbf{H}_{s}-\mathbf{M}_{h}^{n+\frac{1}{2}}\right|^{2}
$$

we have established that, $\forall n \geq 1$,

$$
U_{h}\left(n+\frac{1}{2}\right)-U_{h}\left(n-\frac{1}{2}\right)=-2 \frac{\alpha}{|\gamma|} \mu_{0}\left|\mathbf{M}_{h}^{n+\frac{1}{2}}-\mathbf{M}_{h}^{n-\frac{1}{2}}\right|^{2}
$$


This shows that the discrete quantity $U_{h}\left(n+\frac{1}{2}\right)$ decreases at each step of the computation. We know that $2 \mu_{0} K\left|P\left(\mathbf{M}_{h}^{n+\frac{1}{2}}\right)\right|^{2}+\mu_{0}\left|\mathbf{H}_{s}-\mathbf{M}_{h}^{n+\frac{1}{2}}\right|^{2}$ is a positive term. What can we conclude about the quantity $\hat{U}_{h}\left(n+\frac{1}{2}\right)=$ $\left\|\mathbf{H}_{h}^{n+\frac{1}{2}}\right\|^{2}+\left(\mathbf{E}_{h}^{n}, \mathbf{E}_{h}^{n+1}\right)$ ? Using the fact that

$$
\left(\mathbf{E}_{h}^{n}, \mathbf{E}_{h}^{n+1}\right)=\left\|\frac{\mathbf{E}_{h}^{n+1}+\mathbf{E}_{h}^{n}}{2}\right\|^{2}-\frac{\Delta t^{2}}{4}\left\|\frac{\mathbf{E}_{h}^{n+1}-\mathbf{E}_{h}^{n}}{\Delta t}\right\|^{2}=\left\|\frac{\mathbf{E}_{h}^{n+1}+\mathbf{E}_{h}^{n}}{2}\right\|^{2}-\frac{\Delta t^{2}}{4}\left\|\operatorname{curl}_{h} \mathbf{H}_{h}^{n+\frac{1}{2}}\right\|^{2}
$$

and the fact that $\left\|\operatorname{curl}_{h} \mathbf{H}_{h}^{n+\frac{1}{2}}\right\| \leq \frac{4}{h^{2}}\left\|\mathbf{H}_{h}^{n+\frac{1}{2}}\right\|^{2}$, we see that

$$
\hat{U}_{h}\left(n+\frac{1}{2}\right) \geq\left(1-\frac{\Delta t^{2}}{h^{2}}\right)\left\|\mathbf{H}_{h}^{n+\frac{1}{2}}\right\|^{2}+\left\|\frac{\mathbf{E}_{h}^{n}+\mathbf{E}_{h}^{n+1}}{2}\right\|^{2}
$$

and we can conclude that $\hat{U}_{h}\left(n+\frac{1}{2}\right)$ is positive, and therefore that the scheme is stable, under the classical CFL condition: $\frac{\Delta t}{h} \leq 1$.

\section{NUMERICAL EXPERIMENTS}

In this last part, we perform some numerical simulations. The results that we present are not really "discussed"; our goal consists only in illustrating our numerical method.

First of all we consider the propagation of a sustained signal inside a homogeneous domain: a vacuum first, and then a ferromagnetic domain. We represent only one component of the magnetic field $\mathbf{H}$ at two different times. (In every case, we use transparent boundary conditions, namely $\mathbf{E} \times \mathbf{e}_{x} \pm \mathbf{e}_{x} \times\left(\mathbf{H} \times \mathbf{e}_{x}\right)=0$.) See Figure 1.

In the second case $(\mathbf{M} \neq 0)$, we see the exponential decay of the propagating signal. After some time, a steady state will be reached.

We show now some scattering experiments. The domain of calculation is divided into three parts: on the left and the right sides we consider a vacuum $(\mathbf{M}=0)$, in the center of the calculus domain, a ferromagnetic material. Six snapshots of one component of the magnetic field $\mathbf{H}$ are represented. See Figure 2.

At time $t_{3}$ and $t_{4}$, we see propagating toward the left a reflected part of the signal due to the interface, while a transmitted part is decreasing inside the ferromagnetic media. At time $t_{5}$, the transmitted part of the signal reaches the vacuum on the right side.

\section{Appendix A}

We have shown in Section 2.5 how to get $H^{1}$ estimates of our local solutions to guarantee that they are indeed maximal solutions. In this appendix, we show that the computations of Step 3 of our proof are still valid in the case $\left(\alpha \neq 0, \mathbf{H}_{\text {eff }}(\mathbf{M}) \neq 0\right)$.

In other words, we work now from the complete (LLG) equation

$$
\frac{\partial \mathbf{M}}{\partial t}=\mathbf{H}_{T}(\mathbf{H}, \mathbf{M}) \times \mathbf{M}+\frac{\alpha}{|\mathbf{M}|} \mathbf{M} \times\left(\mathbf{H}_{T}(\mathbf{H}, \mathbf{M}) \times \mathbf{M}\right)
$$



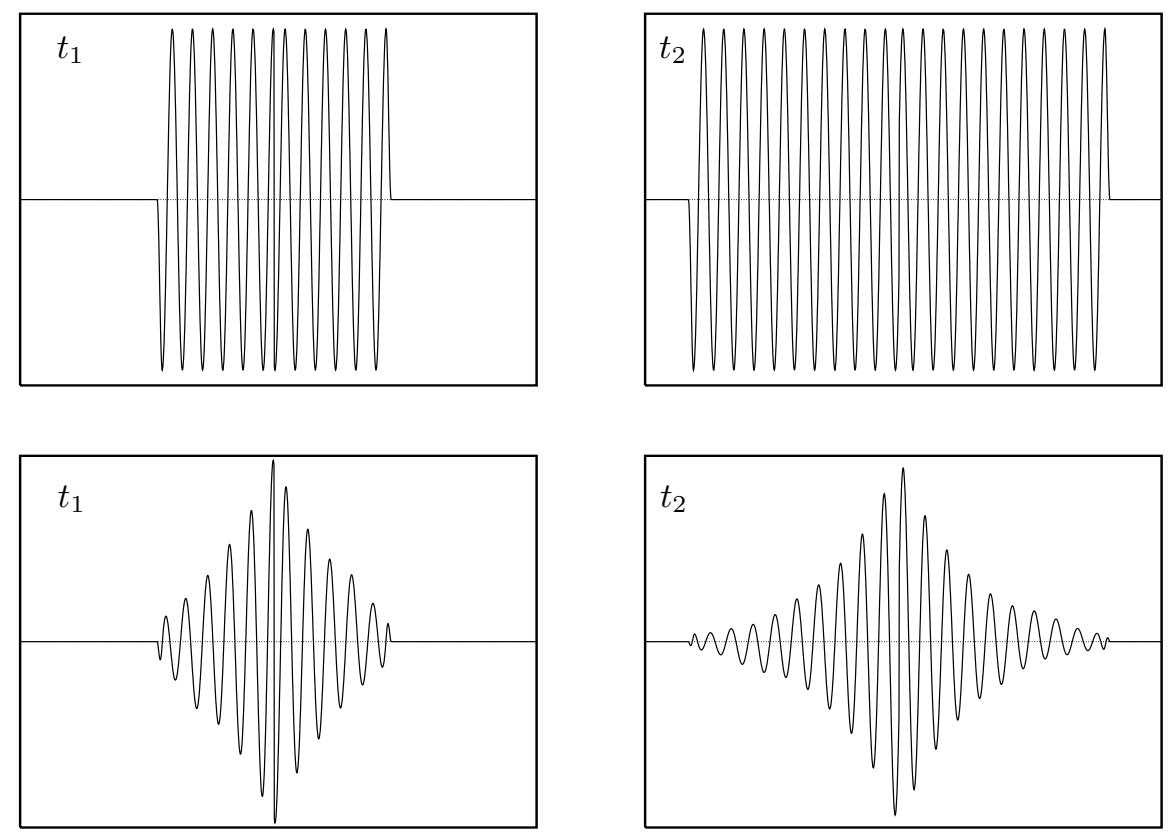

Figure 1. $h=4 \times 10^{-4} \mathrm{~m}, \Delta t=1.33 \times 10^{-4} \mathrm{~s}, f=13 \mathrm{GHz}, H_{s}=10^{5} \mathrm{~A} / \mathrm{m},\left|\mathbf{M}_{0}\right|=$ 0 and $10^{5} \mathrm{~A} / \mathrm{m}, K=1 \mathrm{SI}$.

and we want to get again $H^{1}$ estimates from (74):

$$
\frac{1}{2}\left(\left\|\frac{\partial \mathbf{H}}{\partial t}\right\|_{L^{2}}^{2}+\|\operatorname{curl} \mathbf{H}\|_{L^{2}}^{2}\right)=\frac{1}{2}\left(\left\|\operatorname{curl} \mathbf{H}_{0}\right\|_{L^{2}}^{2}+\|\Lambda(0)\|_{L^{2}}^{2}\right)-\int_{0}^{t} \int_{\mathbb{R}} \frac{\partial^{2} \mathbf{M}}{\partial t^{2}} \cdot \frac{\partial \mathbf{H}}{\partial t} \mathrm{~d} x \mathrm{~d} s .
$$

First, let us recall that $\mathbf{H}_{\text {eff }}(\mathbf{M})$ is affine with respect to $\mathbf{M}$ which yields to:

$$
\exists C_{1}, \quad\left\|\mathbf{H}_{\mathrm{eff}}\right\|_{L^{\infty}} \leq\left\|\mathbf{H}_{s}\right\|_{L^{\infty}}+C_{1}\|\mathbf{M}\|_{L^{\infty}} \quad \text { and } \quad\left|\frac{\partial \mathbf{H}_{\mathrm{eff}}}{\partial t}\right| \leq C_{1}\left|\frac{\partial \mathbf{M}}{\partial t}\right| .
$$

We shall use in the forthcoming computations the fact that the (LLG) equation ensures that

$$
\left|\frac{\partial \mathbf{M}}{\partial t}\right| \leq(1+\alpha)|\mathbf{M}|\left|\mathbf{H}_{T}(\mathbf{H}, \mathbf{M})\right|
$$

and also a generalized Gronwall type lemma.

Lemma 6. Given two positive constants $C$ and $C^{\prime}$ and a positive continuous function $G(t)$ on $[0, T]$ such that, $\forall t \in[0, T]$,

$$
G(t) \leq G(0)+C^{\prime} t+C \int_{0}^{t} G(s) \mathrm{d} s
$$

Then

$$
G(t) \leq G(0) \mathrm{e}^{C t}+\frac{C^{\prime}}{C}\left(\mathrm{e}^{C t}-1\right)
$$



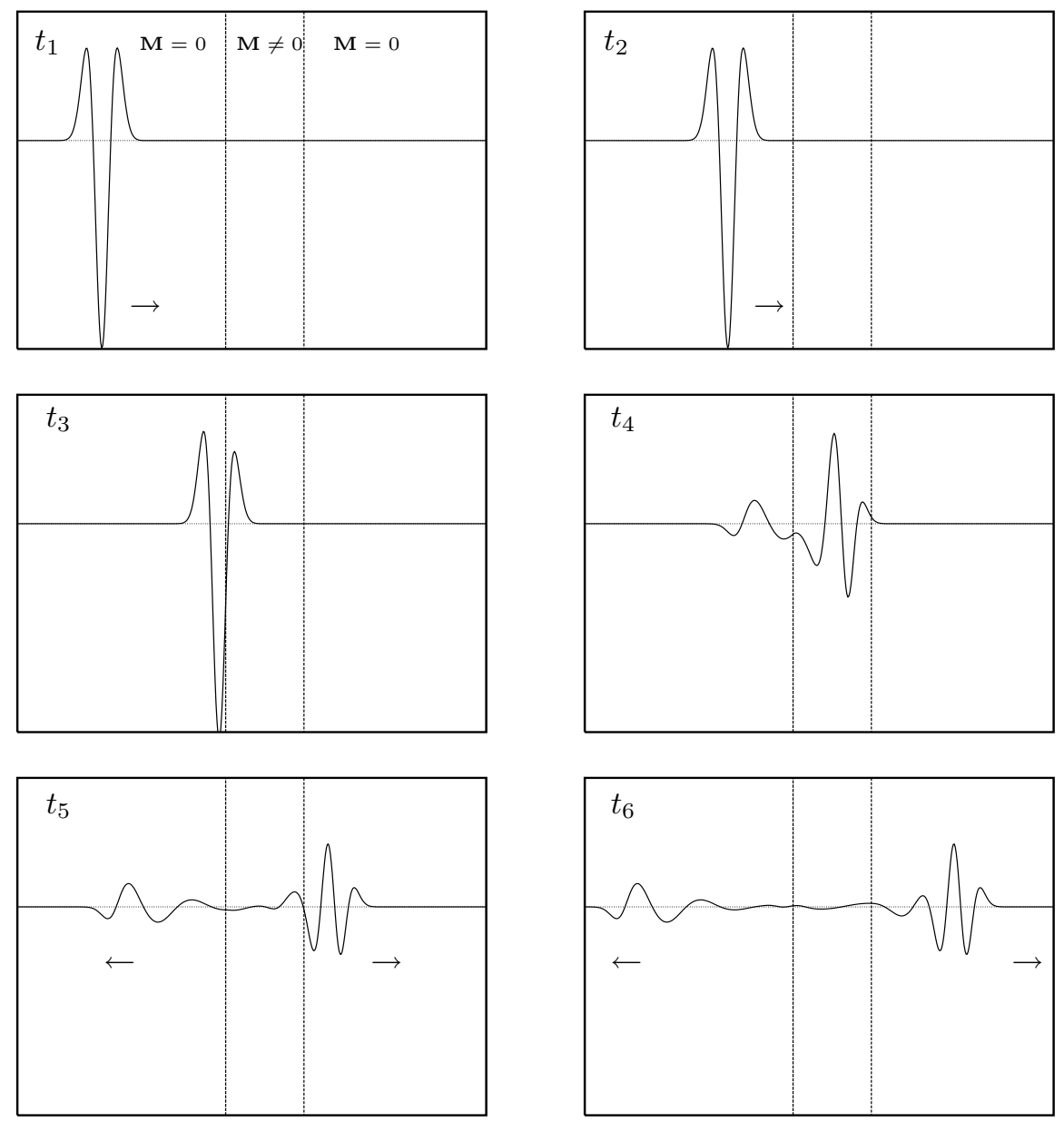

Figure 2. $h=7,5 \times 10^{-4} \mathrm{~m}, \Delta t=2,5 \times 10^{-4} \mathrm{~s}, f=13 \mathrm{GHz}, H_{s}=10^{5} \mathrm{~A} / \mathrm{m},\left|\mathbf{M}_{0}\right|=$ $10^{5} \mathrm{~A} / \mathrm{m}, K=1 \mathrm{SI}$.

Proof. We introduce the differentiable function $\phi(t)=G(0)+C^{\prime} t+C \int_{0}^{t} G(s) \mathrm{d} s$. We have

$$
\phi^{\prime}(t)=C^{\prime}+C G(t) \leq C^{\prime}+C \phi(t)
$$

and we deduce that $\frac{C \phi^{\prime}(t)}{C^{\prime}+C \phi(t)} \leq C$. This result is then integrated in time to give

$$
\frac{C^{\prime}}{C}+\phi(t) \leq\left(\frac{C^{\prime}}{C}+\phi(0)\right) \mathrm{e}^{C t}
$$

Hence the result, given that $G(t) \leq \phi(t)$ and $G(0)=\phi(0)$. 
Taking the derivative in time of the (LLG) equation, we can compute at each point:

$$
\begin{aligned}
\frac{\partial^{2} \mathbf{M}}{\partial t^{2}}= & \frac{\partial \mathbf{H}_{T}}{\partial t} \times \mathbf{M}+\mathbf{H}_{T} \times \frac{\partial \mathbf{M}}{\partial t}+\frac{\alpha}{|\mathbf{M}|} \frac{\partial \mathbf{M}}{\partial t} \times\left(\mathbf{H}_{T} \times \mathbf{M}\right) \\
& +\frac{\alpha}{|\mathbf{M}|} \mathbf{M} \times\left(\frac{\partial \mathbf{H}_{T}}{\partial t} \times \mathbf{M}\right)+\frac{\alpha}{|\mathbf{M}|} \mathbf{M} \times\left(\mathbf{H}_{T} \times \frac{\partial \mathbf{M}}{\partial t}\right)
\end{aligned}
$$

or, by eliminating $\frac{\partial \mathbf{M}}{\partial t}$ with the (LLG) equation,

$$
\begin{aligned}
\frac{\partial^{2} \mathbf{M}}{\partial t^{2}}= & \frac{\partial \mathbf{H}_{T}}{\partial t} \times \mathbf{M}+\mathbf{H}_{T} \times\left(\mathbf{H}_{T} \times \mathbf{M}\right)+\frac{\alpha}{|\mathbf{M}|} \mathbf{H}_{T} \times\left[\mathbf{M} \times\left(\mathbf{H}_{T} \times \mathbf{M}\right)\right] \\
& +\left(\frac{\alpha}{|\mathbf{M}|}\right)^{2}\left[\mathbf{M} \times\left(\mathbf{H}_{T} \times \mathbf{M}\right)\right] \times\left(\mathbf{H}_{T} \times \mathbf{M}\right)+\frac{\alpha}{|\mathbf{M}|} \mathbf{M} \times\left(\frac{\partial \mathbf{H}_{T}}{\partial t} \times \mathbf{M}\right) \\
& +\frac{\alpha}{|\mathbf{M}|} \mathbf{M} \times\left[\mathbf{H}_{T} \times\left(\mathbf{H}_{T} \times \mathbf{M}\right)\right]+\left(\frac{\alpha}{|\mathbf{M}|}\right)^{2} \mathbf{M} \times\left[\mathbf{H}_{T} \times\left(\mathbf{M} \times\left(\mathbf{H}_{T} \times \mathbf{M}\right)\right)\right] .
\end{aligned}
$$

Then we can take the scalar product with $\frac{\partial \mathbf{H}}{\partial t}$ :

$$
\begin{aligned}
\frac{\partial^{2} \mathbf{M}}{\partial t^{2}} \cdot \frac{\partial \mathbf{H}}{\partial t}= & \left\{\mathbf{H}_{T} \times\left(\mathbf{H}_{T} \times \mathbf{M}\right)+\frac{\alpha}{|\mathbf{M}|} \mathbf{H}_{T} \times\left[\mathbf{M} \times\left(\mathbf{H}_{T} \times \mathbf{M}\right)\right]\right. \\
& +\frac{\alpha^{2}}{|\mathbf{M}|^{2}}\left[\mathbf{M} \times\left(\mathbf{H}_{T} \times \mathbf{M}\right)\right] \times\left(\mathbf{H}_{T} \times \mathbf{M}\right)+\frac{\alpha}{|\mathbf{M}|} \mathbf{M} \times\left[\mathbf{H}_{T} \times\left(\mathbf{H}_{T} \times \mathbf{M}\right)\right] \\
& \left.+\frac{\alpha^{2}}{|\mathbf{M}|^{2}} \mathbf{M} \times\left[\mathbf{H}_{T} \times\left(\mathbf{M} \times\left(\mathbf{H}_{T} \times \mathbf{M}\right)\right)\right]\right\} \cdot \frac{\partial \mathbf{H}}{\partial t}+\left(\frac{\partial \mathbf{H}_{T}}{\partial t}, \mathbf{M}, \frac{\partial \mathbf{H}}{\partial t}\right) \\
& +\frac{\alpha}{|\mathbf{M}|}\left(\mathbf{M}, \frac{\partial \mathbf{H}_{T}}{\partial t} \times \mathbf{M}, \frac{\partial \mathbf{H}}{\partial t}\right) \\
= & T_{1}+T_{2}+T_{3},
\end{aligned}
$$

where

$$
\begin{aligned}
T_{1}= & \left\{\mathbf{H}_{T} \times\left(\mathbf{H}_{T} \times \mathbf{M}\right)+\frac{\alpha}{|\mathbf{M}|} \mathbf{H}_{T} \times\left[\mathbf{M} \times\left(\mathbf{H}_{T} \times \mathbf{M}\right)\right]+\frac{\alpha}{|\mathbf{M}|}\left(\mathbf{M}, \frac{\partial \mathbf{H}_{T}}{\partial t} \times \mathbf{M}, \frac{\partial \mathbf{H}}{\partial t}\right)\right. \\
& \left.+\frac{\alpha}{|\mathbf{M}|} \mathbf{M} \times\left[\mathbf{H}_{T} \times\left(\mathbf{H}_{T} \times \mathbf{M}\right)\right]+\frac{\alpha^{2}}{|\mathbf{M}|^{2}} \mathbf{M} \times\left[\mathbf{H}_{T} \times\left(\mathbf{M} \times\left(\mathbf{H}_{T} \times \mathbf{M}\right)\right)\right]\right\} \cdot \frac{\partial \mathbf{H}}{\partial t}
\end{aligned}
$$

$T_{2}=\left(\frac{\partial \mathbf{H}_{T}}{\partial t}, \mathbf{M}, \frac{\partial \mathbf{H}}{\partial t}\right)$ and $T_{3}=\frac{\alpha}{|\mathbf{M}|}\left(\mathbf{M}, \frac{\partial \mathbf{H}_{T}}{\partial t} \times \mathbf{M}, \frac{\partial \mathbf{H}}{\partial t}\right)$. Each term can be bounded:

1. Using Cauchy-Schwartz inequalities, we have

$$
T_{1} \leq 2\left(1+\alpha+\alpha^{2}\right)|\mathbf{M}|\left|\mathbf{H}_{T}\right|^{2}\left|\frac{\partial \mathbf{H}}{\partial t}\right| \leq\left(1+\alpha+\alpha^{2}\right)|\mathbf{M}|\left(|\mathbf{H}|^{2}+\left|\mathbf{H}_{\mathrm{eff}}\right|^{2}\right)\left|\frac{\partial \mathbf{H}}{\partial t}\right|
$$

and then, with (161), we get

$$
\int_{\mathbb{R}} T_{1} \mathrm{~d} x \leq 2\left(1+\alpha+\alpha^{2}\right)\left[\|\mathbf{M}\|_{L^{\infty}} \int_{\mathbb{R}}|\mathbf{H}|^{2}\left|\frac{\partial \mathbf{H}}{\partial t}\right| \mathrm{d} x+\left(\left\|\mathbf{H}_{s}\right\|_{L^{\infty}}+C_{1}\|\mathbf{M}\|_{L^{\infty}}\right)^{2} \int_{\mathbb{R}}|\mathbf{M}|\left|\frac{\partial \mathbf{H}}{\partial t}\right| \mathrm{d} x\right] .
$$


2. As $\left(\frac{\partial \mathbf{H}_{T}}{\partial t}, \mathbf{M}, \frac{\partial \mathbf{H}}{\partial t}\right)=\left(\frac{\partial \mathbf{H}_{\mathrm{eff}}}{\partial t}, \mathbf{M}, \frac{\partial \mathbf{H}}{\partial t}\right)$, using (161) and (162), we have

$$
T_{2} \leq C_{1}|\mathbf{M}|\left|\frac{\partial \mathbf{M}}{\partial t}\right|\left|\frac{\partial \mathbf{H}}{\partial t}\right| \leq C_{1}(1+\alpha)|\mathbf{M}|^{2}|\mathbf{H}|\left|\frac{\partial \mathbf{H}}{\partial t}\right|
$$

Thus

$$
\int_{\mathbb{R}} T_{2} \mathrm{~d} x \leq C_{1}(1+\alpha)\|\mathbf{M}\|_{L^{\infty}}^{2} \int_{\mathbb{R}}\left|\mathbf{H}_{T}\right|\left|\frac{\partial \mathbf{H}}{\partial t}\right| \mathrm{d} x
$$

3. As $T_{3}=\frac{\alpha}{|\mathbf{M}|}\left(\left|\frac{\partial \mathbf{H}}{\partial t} \times \mathbf{M}\right|^{2}+\left(\frac{\partial \mathbf{H}_{\mathrm{eff}}}{\partial t} \times \mathbf{M}\right) \cdot\left(\frac{\partial \mathbf{H}}{\partial t} \times \mathbf{M}\right)\right)$, we have with (161) and (162)

$$
\int_{\mathbb{R}} T_{3} \mathrm{~d} x \leq \alpha\|\mathbf{M}\|_{L^{\infty}}\left\|\frac{\partial \mathbf{H}}{\partial t}\right\|_{L^{2}}^{2}+\alpha(1+\alpha)\|\mathbf{M}\|_{L^{\infty}}^{2} \int_{\mathbb{R}}\left|\mathbf{H}_{T}\right|\left|\frac{\partial \mathbf{H}}{\partial t}\right| \mathrm{d} x .
$$

Then, if we define $C_{2}$ by

$$
C_{2}=\operatorname{Max}\left\{\left(1+\alpha+\alpha^{2}\right)\left(\left\|\mathbf{H}_{s}\right\|_{L^{\infty}}+C_{1}\|\mathbf{M}\|_{L^{\infty}}\right), C_{1}\left(1+\alpha^{2}\right)\|\mathbf{M}\|_{L^{\infty}}^{2}\right\}
$$

we can add (168), (170) and (171) to obtain

$$
\int_{\mathbb{R}} \frac{\partial^{2} \mathbf{M}}{\partial t^{2}} \cdot \frac{\partial \mathbf{H}}{\partial t} \mathrm{~d} x \leq 3 C_{2}\left[\int_{\mathbb{R}}|\mathbf{H}|^{2}\left|\frac{\partial \mathbf{H}}{\partial t}\right| \mathrm{d} x+\|\mathbf{M}\|_{L^{2}}^{2}+\|\mathbf{H}\|_{L^{2}}^{2}+\left\|\frac{\partial \mathbf{H}}{\partial t}\right\|_{L^{2}}^{2}\right] .
$$

The last term to integrate is exactly the one that we have considered in Section 2.5 in (87)

$$
\int_{\mathbb{R}}|\mathbf{H}|^{2}\left|\frac{\partial \mathbf{H}}{\partial t}\right| \mathrm{d} x \leq 2\left(2 \mathcal{E}_{0}\right)^{3 / 4}\|\operatorname{curl} \mathbf{H}\|_{L^{2}}^{1 / 2}\left\|\frac{\partial \mathbf{H}}{\partial t}\right\|_{L^{2}} .
$$

Using (90), we see that this is equivalent to

$$
\int_{\mathbb{R}}|\mathbf{H}|^{2}\left|\frac{\partial \mathbf{H}}{\partial t}\right| \mathrm{d} x \leq\left(2 \mathcal{E}_{0}\right)^{3 / 4}\left(1+\|\operatorname{curl} \mathbf{H}\|_{L^{2}}^{2}+\left\|\frac{\partial \mathbf{H}}{\partial t}\right\|_{L^{2}}^{2}\right) .
$$

If we let $C=\left(1+\mathcal{E}_{0}\right)\left(1+C_{2}\right)$, we can deduce from (74) that, $\forall t \in[0, T]$,

$$
\begin{aligned}
\frac{1}{2}\left(\left\|\frac{\partial \mathbf{H}}{\partial t}(t)\right\|_{L^{2}}^{2}+\right. & \left.\|\operatorname{curl} \mathbf{H}(t)\|_{L^{2}}^{2}\right) \leq \frac{1}{2}\left(\left\|\operatorname{curl} \mathbf{H}_{0}\right\|_{L^{2}}^{2}+\|\Lambda(0)\|_{L^{2}}^{2}\right) \\
& +C\left(1+\left\|\mathbf{H}_{0}\right\|_{L^{2}}^{2}+\left\|\mathbf{M}_{0}\right\|_{L^{2}}^{2}\right) t+C \int_{0}^{t}\left(\|\operatorname{curl} \mathbf{H}\|_{L^{2}}^{2}+\left\|\frac{\partial \mathbf{H}}{\partial t}\right\|_{L^{2}}^{2}\right) \mathrm{d} s
\end{aligned}
$$

This is equivalent to

$$
G(t) \leq G(0)+C^{\prime} t+C \int_{0}^{t} G(s) \mathrm{d} s
$$


where $G(t)=\left(\|\operatorname{curl} \mathbf{H}(t)\|_{L^{2}}^{2}+\left\|\frac{\partial \mathbf{H}}{\partial t}(t)\right\|_{L^{2}}^{2}\right)$ and $C^{\prime}=C\left(1+\left\|\mathbf{H}_{0}\right\|_{L^{2}}^{2}+\left\|\mathbf{M}_{0}\right\|_{L^{2}}^{2}\right)$. Applying Lemma 6, we get the desired estimate:

$$
\left(\|\operatorname{curl} \mathbf{H}(t)\|_{L^{2}}^{2}+\left\|\frac{\partial \mathbf{H}}{\partial t}(t)\right\|_{L^{2}}^{2}\right) \leq\left(\|\operatorname{curl} \mathbf{H}(0)\|_{L^{2}}^{2}+\left\|\frac{\partial \mathbf{H}}{\partial t}(0)\right\|_{L^{2}}^{2}\right) \mathrm{e}^{C t}+\frac{C^{\prime}}{C}\left(\mathrm{e}^{C t}-1\right) .
$$

This is the equivalent of $(93)$ for the case $\left(\alpha \neq 0, \mathbf{H}_{\text {eff }}(\mathbf{M}) \neq 0\right)$.

\section{REFERENCES}

[1] A. Bagneres, Simulation de la structure de parois dans un matériau magnétique. Ph.D. thesis, Institut National Polytechnique de Grenoble (1988).

[2] W.B. Brown, Micromagnetics. Interscience Publisher (1963).

[3] B. Lax and K.J. Button, Microwave ferrites and ferromagnetics. McGraw-Hill book company (1962).

[4] G. Pircher, Ferrites et grenats, phénomènes non linéaires. Dunod (1970).

[5] A. Visintin, On Landau-Lifchitz' equations for ferromagnetism. Jap. J. Appl. Math. 2 (1985) 69-84.

[6] A. Haraux, Nonlinear evolution equations - global behavior of solutions. Springer-Verlag (1981).

[7] A. Pazy, Semigroups of linear operators and applications to partial differential equations. New York-Berlin-Tokyo, Springer (1983).

[8] P. Monk and A. Parrot, A dispersion analysis of finite element methods for Maxwell's equations. SIAM J. Sci. Comp. 15 (1994) 916-937.

[9] K.S. Yee, Numerical solution of initial boundary value problems involving Maxwell's equations. IEEE Trans. Antennas Propag. 14 (1966) 302-307.

[10] J. Pereda, L. Vielva, M. Solano, A. Vegas and A. Prieto, FDTD analysis of magnetized ferrites: application to the calculation of dispersion characteristics of ferrite-loaded waveguides. IEEE Trans. Microwave Theory Tech. 43 (1995) 350-356.

[11] J. Pereda, L. Vielva, A. Vegas and A. Prieto, A treatment of magnetized ferrites using the FDTD method. Microwave and guided wave letters 3 (1993) 136-138.

[12] J.F. Lee and R. Mittra, Analysis of microwave ferrite devices by using the finite element method. J. Appl. Phys. 69 (1991) 5032-5034.

[13] A. Reinex, T. Monediere and F. Jecko, Ferrite analysis using the finite-difference time-domain method. Microwave and optical technology letters 5 (1992) 685-686.

[14] T. Monediere, M. Latrach and F. Jecko, Resonant modes and magnetic losses in a ferrite coaxial resonator. Journal of electromagnetic waves and applications 6 (1992) 199-217.

[15] G. Zheng and K. Chen, Nonlinear study of microstrip lines containing ferrite dielectric layers. Int. J. Infrared Millim. Waves 13 (1992) 1115-1125.

[16] P. Joly and O. Vacus, Numerical simulation of electromagnetic wave propagation in 2D non-linear ferromagnetic materials (in preparation).

[17] P. Joly and O. Vacus, Propagation d'ondes en milieu ferromagnétique 1D : existence et unicité de solutions faibles. C.R. Acad. Sci. (submitted). 